\title{
Representation and application of Fuzzy soft sets in type-2 environment
}

\author{
Biplab Paik ${ }^{1}$ (1) $\cdot$ Shyamal Kumar Mondal ${ }^{1}$
}

Received: 12 February 2020 / Accepted: 25 January 2021 / Published online: 19 February 2021

(c) The Author(s) 2021

\begin{abstract}
This paper has represented a soft-set in the type-2 environment by its simplest form as an augmentation to soft-set theories. Furthermore, we have applied the type-2 fuzzy soft set(T2FSS) by using our most straightforward representation to find the solution of a decision-making-problem (DMP) based-on T2FSS as well as weighted type-2 fuzzy soft set (WT2FSS). We have proposed two definitions, namely, Mid- $\alpha$-threshold fuzzy-set of a T2FSS and Mid- $\lambda$-threshold fuzzy-set of a T2FSS. Furthermore, we have presented the definition, namely, level fuzzy-soft-set(LFSS) of a T2FSS. Using this concept, we have prepared two algorithms to select one object in T2FSS as well as WT2FSS based on DMP, which take regret disinclination and expectation preference of decision-makers into consideration in the decision procedure. Also, we have presented two numerical examples at the end.
\end{abstract}

Keywords Fuzzy set · Fuzzy soft set · Type-2 fuzzy soft set · Weighted type-2 fuzzy soft set · Decision analysis

\section{Introduction}

Soft set theory [1] introduced by Molodtsov is an essential tool for operating with uncertainties. Compared with some traditional mathematical tools for dealing with uncertainties, such as the theory of probability, the idea of fuzzy sets [2], and the theory of rough sets, the advantage of soft set theory is that it is free from the inadequacy of the parametrization tools of those theories. It has been exhibited that soft-set theory provokes an excellent dynamical for applications in multiple fields such as Riemann integration, smoothness of functions, DMP, the theory of measurement, game theory, etc.

The Soft-set theory has accepted considerable attention. Kong et al. [3] proposed the idea of average parameter reduction into soft sets. Ali et al. [4] defined many operations in soft-set theory. Zou et al. [5] invented data analysis techniques for soft-sets with incomplete pieces of information.

Biplab Paik

biplabmath@gmail.com

Shyamal Kumar Mondal

shyamal_260180@yahoo.com

1 Department of Applied Mathematics with Oceanology and Computer Programming, Vidyasagar University, Midnapore, W.B. 721 102, India
Cagman et al. [6] suggested, differently, some soft-sets operations and formed a uni-int decision-making method. Herawan and Deris [7] gave a soft-set technique for rules of minimal as well as maximal association rules from transnational data sets. Gong et al. [8] introduced the idea of a bijective soft-set and described the operations on soft-sets.

These researches are founded on the ordinary soft-set theory. It is possible to combine Soft-set theory with other mathematical theories. Fuzzy-soft-set(FSS) is an extension of the classical soft-set. Besides, researchers have extended soft set theory to different environments. Fuzzy soft set theory $[9,10]$, intuitionistic fuzzy soft set theory $[11,12]$, neutrosophic soft set theory $[13,14]$, vague soft set [15], rough soft sets [16], trapezoidal interval type-2 fuzzy soft set theory [17], etc. are some important extensions of soft set theory. Then, several types of decision-making have been disposed by using soft set theory. For example, Manna et al. developed an approach to linguistic valued soft multi-criteria group decision-making problems [18]. Maji et al. [19] focused on introducing the intuitionistic fuzzy sets in soft set theory [20]. By integrating the interval-valued intuitionistic fuzzy sets with soft sets, Jiang et al. [21] defined a new soft set model. Several modeling applications [22,23] have achieved better performance system analysis applied to a number of very different applications. Pozna et al. [23] used fuzzy signatures to develop an expert system modeling and presented

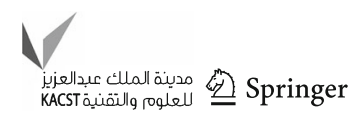


a convenient framework for the symbolic representation of data.

Crisp membership functions characterize ordinary type1 fuzzy sets. Zadeh [24] described the Type-2 fuzzy set in which the membership function maps into an FS in the interval $[0,1]$. We can find the type- 2 fuzzy set's preliminary ideas and some real applications in [25-31]. In the beginning, researchers were focusing on Type-1 fuzzy logic and its applications, but as the new challenging problems arrived, it was natural to shift the interest of people towards an approach that could handle more complex situations. This is how we have reached today the state of the type-2 fuzzy logic area. Type-2 fuzzy logic, which is an extended version or generalization of the type-1 fuzzy logic, can provide a significant level of imprecision modeling [32]. Thus, type-2 fuzzy logic offers capabilities to handle linguistic related uncertainties by considering the unreliability and vagueness of information [33]. In their work, Karnik and Mendel suggested that type-2 fuzzy sets have a certain degree of uncertainty associated with their membership function [34]. A type-2 fuzzy set is the principle idea of type- 2 fuzzy logic. The type- 2 fuzzy set and type2 fuzzy logic have accepted considerable attention. Hagras [35] proposed a hierarchical type-2 fuzzy logic control architecture for autonomous mobile robots. Karnik et al. [25] suggested some applications on type- 2 fuzzy sets. They also applied [26] type-2 fuzzy logic systems to the forecasting of time-series. Mizumoto et al. [29] researched on type-2 fuzzy set under the algebraic product and algebraic sum. Attribute reduction plays a vital role in decision-making problems. Wu et al. [30] proposed an interval type-2 fuzzy rough set model for attribute reduction. Zeng et al. [31] suggested type-2 fuzzy hidden Markov models and their application to speech recognition. Mendel [36] proposed a high-level retrospective of type-2 fuzzy sets and fuzzy logic systems. Lathamaheswari et al. [37] studied the application of triangular interval type-2 fuzzy soft sets. Melin and Castillo [38] presented a brief review of type- 2 fuzzy logic applications in various fields.

In practical applications, we are often faced with the situation in which the evaluation of parameters is a fuzzy concept. For example, when we are going to buy a shirt, we need to consider the shirt's attractiveness. We can provide some linguistic terms, such as good, medium, bad, etc., to evaluation the shirt's attractiveness. Here good, medium, bad etc are fuzzy concepts, and they can be represented by fuzzy sets rather than exact numerical values. It is difficult for the classical soft set and its existing extensions to deal with the above case because evaluating the object's parameters is a fuzzy concept rather than an exact numerical value. So, it is necessary to extend the soft set theory to accommodate the situations in which the evaluation of parameters is a fuzzy concept. In this regard, Zhang et al. [39] researched the idea of T2FSS by combining the soft set with type- 2 fuzzy set theories. Here, we have represented the T2FSS in the simplest form, and we look into the applications of T2FSS and WT2FSS in DMP. Our contribution to this paper is fourfold:

(1) We are going to represent the T2FSS in the simplest form.

(2) Two definitions, namely, the Mid- $\alpha$-threshold fuzzy-set and Mid- $\lambda$-threshold fuzzy-set for a T2FSS, are proposed. Utilizing these two definitions, we have presented the definition of the level soft set of a T2FSS.

(3) Two algorithms for T2FSS based DMP and WT2FSS based DMP are proposed.

(4) We have picked three real-life problems and solved them using our proposed algorithms.

\section{Definitions}

Definition 1 Fuzzy Set [2] (Type-1 fuzzy-set)

Let $\mathrm{P}$ is the collection of objects denoted generally by $\mathrm{p}$ then a fuzzy set(FS) (Type-1) $F$ in $P$ is a set of ordered pairs: $F=\left\{\left(p, \mu_{F}(p)\right): p \in P\right\}, \mu_{F}(p)$ is called the membership of $p$ in $F$.

Representation of FS (Type-1):

(i) Discrete universe of discourse :

Let $P$ is a collection of objects. $A$ in $P$ is a FS and $\mu_{F}(p)$ is called the grade of membership of $p$ in $F$. Then,

$$
\begin{aligned}
F & =\left\{\left(p, \mu_{F}(p)\right): p \in P\right\} \\
& =\left\{\frac{\mu_{F}\left(p_{1}\right)}{p_{1}}, \frac{\mu_{F}\left(p_{2}\right)}{p_{2}}, \ldots, \frac{\mu_{F}\left(p_{n}\right)}{p_{n}}\right\}
\end{aligned}
$$

(ii) Continuous universe of discourse :

$$
A=\left\{\left(p, \mu_{F}(p)\right): p \in P\right\}=\int_{P} \mu_{F}(p) / p
$$

Example 1 Discrete universe (Fig. 1a)

Let $\mathrm{X}$ be the collections of the number of children in a family such as, $P=\{0,1,2,3,4,5,6\}$

The membership function or the grade of membership given by, $\mu_{A}(0)=0.1, \mu_{A}(1)=0.3, \mu_{A}(2)=$ $0.7, \mu_{A}(3)=1.0, \mu_{A}(4)=0.6, \mu_{A}(5)=0.2, \mu_{A}(6)=$ 0.1 . Then the type- 1 fuzzy set $\mathrm{A}=$ "Sensible number of children" is rewritten by

$$
\begin{aligned}
A & =\left\{\left(p, \mu_{A}(p)\right): p \in P\right\} \\
& =\left\{\frac{0.1}{0}, \frac{0.3}{1}, \frac{0.7}{2}, \frac{1.0}{3}, \frac{0.6}{4}, \frac{0.2}{5}, \frac{0.1}{6}\right\}
\end{aligned}
$$

Example 2 Continuous universe (Fig. 1b) 
Fig. 1 a "Sensible number of children in a family". b"About 50 years old" (a) MF on a Discrete Universe

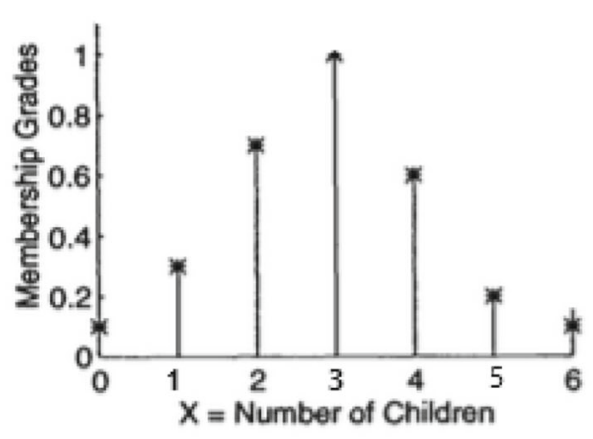

(b) MF on a Continuous Universe

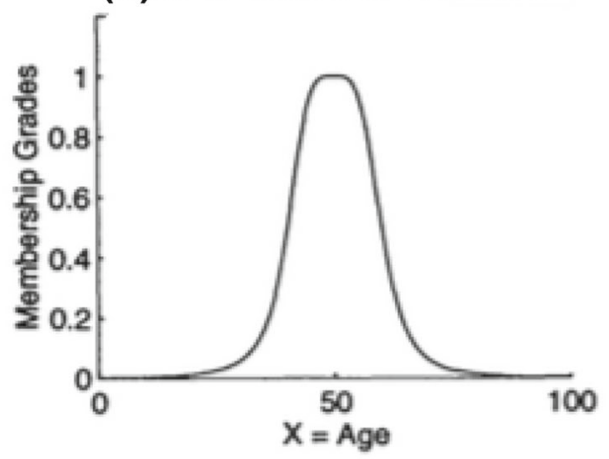

Fig. 2 a "Pictorial representation of a type-2 fuzzy set" b"The secondary memberships for $x=4$ "
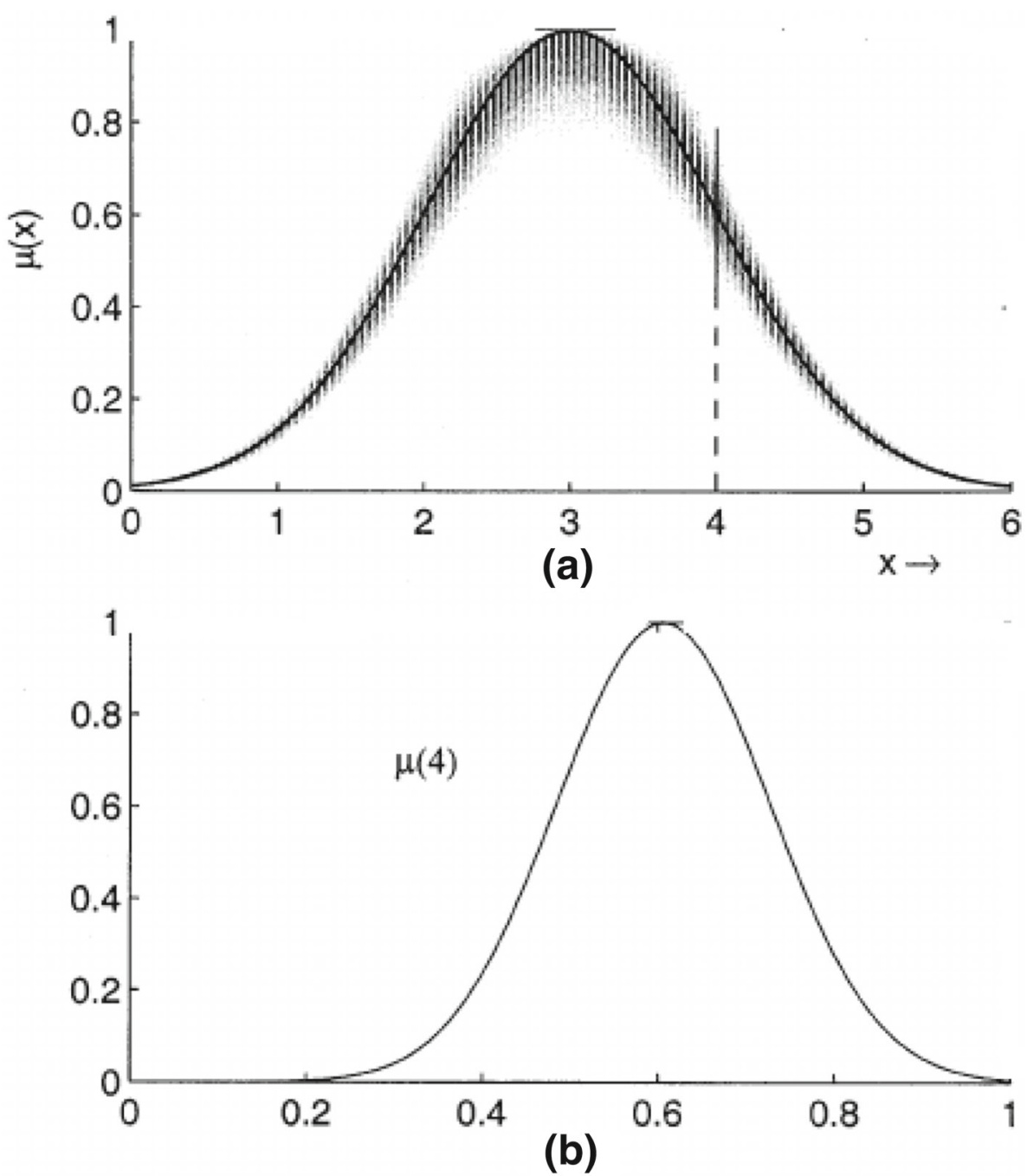

Let $P$ is a collection of different ages. We can consider $P$ as the set of all positive real numbers. That is $P=R^{+}$(Set of positive real numbers).

Let $Q=$ "About 50 years old". Then $Q$ is a type- 1 fuzzy set whose membership function is a continuous function defined by $\mu_{Q}(p)=\frac{1}{1+\left(\frac{p-50}{10}\right)^{4}}$. Then type- 1 fuzzy set also be written by, $Q=\left\{\left(p, \mu_{Q}(p)\right): p \in P\right\}=\int_{P} \mu_{Q}(p) / p$, where $\mu_{Q}(p)=\frac{1}{1+\left(\frac{p-50}{10}\right)^{4}}$.

Definition 2 Type-2 fuzzy set [26]

A type-2 fuzzy-set is a fuzzy-set in $X$ whose membership values are type- 1 fuzzy sets on $[0,1]$. 


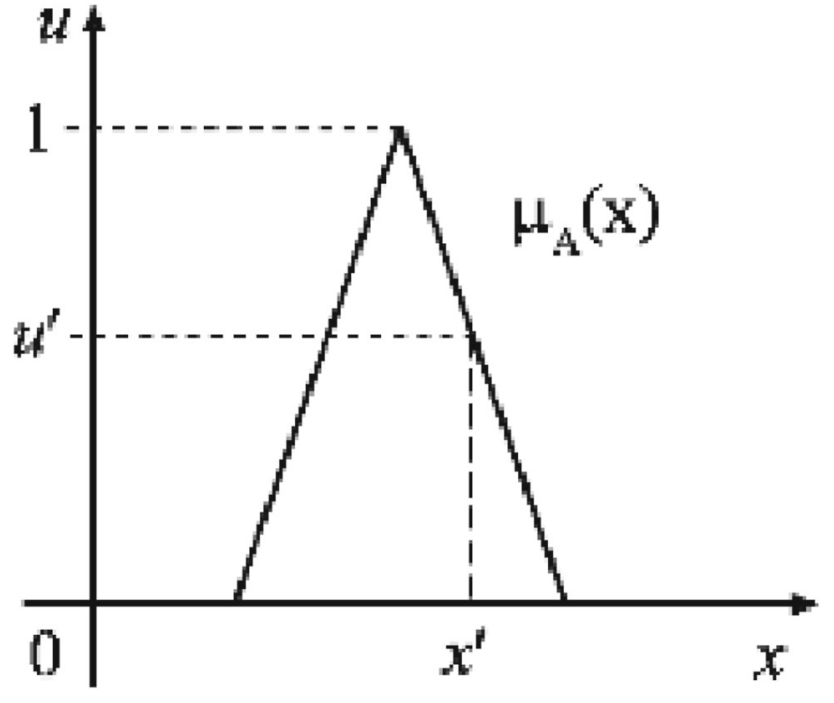

Fig. 3 Type-1 membership function

In this case, the membership values of type- 2 fuzzy-set are secondary membership, and the domain of secondary membership is said to be primary membership.

Example 3 Figure 2a represents a type-2 fuzzy set in which the grade of primary membership for every point in that set is a type- 1 fuzzy set. Also, the figure has shown a shaded region, which is the union of all primary membership grades. We call it the footprint of the uncertainty of the type- 2 membership function (Fig. 2a). The principal membership function, i.e., the set of primary memberships having secondary membership equal to 1 , is given by a thick line. The intensity of the shading is approximately proportional to secondary membership grades. Darker areas indicate more significant secondary memberships. The domain of the membership grade corresponding to $x=4$ is also shown in Fig. 2b.

Example 4 Suppose blurring the type-1 membership function shown in Fig. 3 by moving the trapezium points, either the left or right, in different amounts, as in Fig. 4. Then, at a particular value of $x$, say $x^{\prime}$, there no longer is a single value for the membership function; instead, the membership function takes on values wherever the vertical line intersects the blur. Those values need not all be weighted the same. We create a three-dimensional membership function, called "a type-2 membership function" that characterizes a type-2 fuzzy set, for all $x$.

Definition 3 Type-2 fuzzy soft set [39]

Let $U$ be a universal set and $Q \subseteq P$ be the parameter's set; then $(F, Q)$ is said to be a type-2 fuzzy soft set(T2FSS) over $U$, in which the mapping $F$ is given by $F: Q \rightarrow F_{T 2}(U)$. In another way, a T2FSS is called the parameterized collection of type-2 fuzzy subsets of $U$. For any $e \in Q, F(e)$ is called the

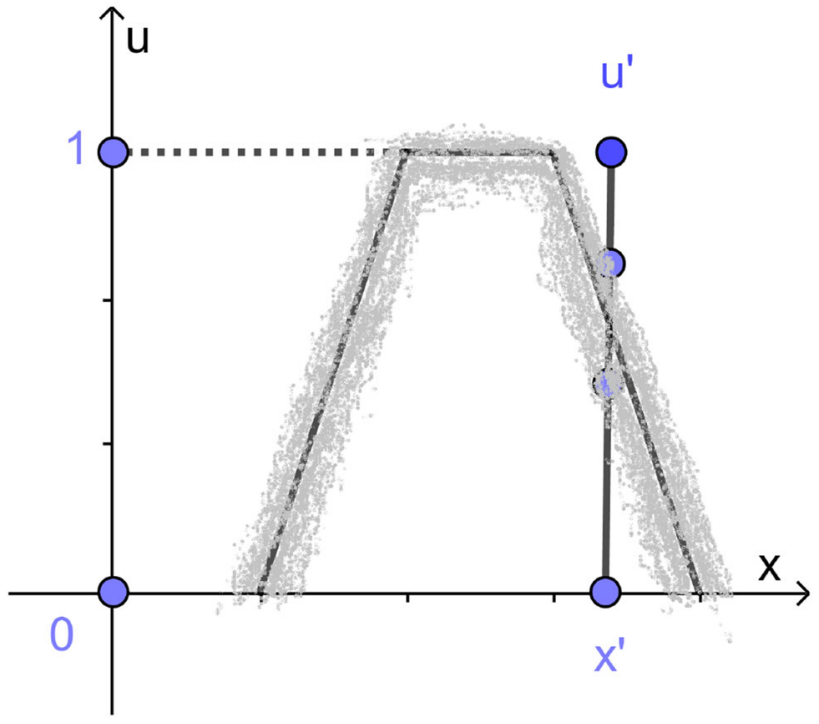

Fig. 4 "Blurred type-1" that is "type-2" membership function, including discretization at $x=x^{\prime}$

set of $e$-approximate elements of the T2FSS $(F, Q)$. Actually it is a type-2 fuzzy set on $U$, and it rewritten as, $F(e)=$ $\int_{p \in U} \int_{v \in J_{p}} \frac{\mu_{F(e)}(p, v)}{(p, v)}=\int_{p \in U} \frac{\left[\int_{v \in J_{p}} f_{p}(v) / v\right]}{p}, J_{p} \subseteq I$. Here, $v, \mu_{F(e)}(p, v)$ are, respectively, the degrees of primary membership and secondary membership that object $\mathrm{p}$ holds on parameter e.

Definition 4 Level soft-set of a type-2 fuzzy-soft-set [39] Let $(\mathcal{F}, Q)$ be a T2FSS on $U$, where $Q \subseteq P$ and $P$ is the set of parameters. Let $\alpha: Q \rightarrow[0,1]$ and $\lambda: Q \rightarrow[0,1]$ be two FS in $Q$. The level soft-set of $(\mathcal{F}, Q)$ with respect to $\alpha$ and $\lambda$ is a crisp soft-set $\mathrm{L}((\mathcal{F}, Q) ; \alpha, \lambda))=\left(\mathcal{F}_{(\alpha, \lambda)}, Q\right)$ defined as follows: for any $a \in Q, \mathcal{F}_{(\alpha, \lambda)}(a)=\mathcal{F}(a)_{\lambda}^{\alpha}=\{p \in U$ : $\left.v(p) \geq \lambda(a), \forall v(p) \in J_{p}^{\alpha}\right\} ;$ where $J_{p}^{\alpha}=\left\{v: \mu_{F(a)}(p, v) \geq\right.$ $\left.\alpha(a), v \in J_{p}\right\}, J_{p} \subseteq I=[0,1], \mu_{F(a)}$ and $v(p)$ are respectively the second membership and first membership of the T2FSS $(\mathcal{F}, Q)$.

\section{Proposed representation of a type-2 fuzzy-set and T2FSS}

In 1965 when Zadeh invented the fuzzy set [2], many criticisms arise that it does not capture the right uncertainty. But that criticism is ended with the invention of the type-2 fuzzy set concept in 1975 by Zadeh [24]. Many researchers work on $[17,25-31,35,37]$ the properties, operations, and applications of type 2 fuzzy sets and its soft set extensions. Here we have represented type 2 fuzzy sets and type 2 fuzzy soft sets in the simplest form and used it in decision-making. 


\section{Representation of a type-2 fuzzy-set}

The type-2 fuzzy set $A$ in $P$ can be represented by $A=$ $\left\{\left(p, \mu_{A}(p)\right): p \in P\right\}=\left\{\left(p,\left(u_{i}, \mu_{u i}(p)\right)\right): p \in\right.$ $\left.P ; u_{i}, \mu_{u i}(p) \in[0,1]\right\}=\left\{\frac{\left(u_{i}, \mu_{u i}\right)}{p}: p \in P ; u_{i}, \mu_{u i} \in\right.$ $[0,1]\}$. Here, $\mathrm{P}$ is the initial universe and $\mu_{A}(p)$ is the secondary membership of the type-2 fuzzy set $A$ in $P$. The domain of secondary membership function $\mu_{A}(p): u_{i} \rightarrow$ $\mu_{u i}(p)$ is $\left\{u_{i}: u_{i} \in[0,1]\right\}$ which is the set of primary membership grades of $A$ in $P$.

Example 5 Let $\mathrm{P}=\{3,4,5,6\}$ and a Type-2 fuzzy-set $\mathrm{Q}$ $=$ "Integers close to 4 ".

Suppose $\left\{u_{i}: u_{i} \in[0,1]\right\}=\{0.5,0.6,0.7,0.8\}$ be the primary membership i.e., the domain of the secondary membership $\mu_{Q}(p)$.

Then type-2 fuzzy set $Q$, defined by $Q=\left\{\left(p, \mu_{Q}(p)\right)\right\}$

where, for $p=3$,

$$
\begin{aligned}
\mu_{Q}(3) & =\left\{\left(u_{i}, \mu_{u i}(3)\right): i=1,2,3,4\right\} \\
& =\{(0.8,0.9),(0.7,0.5),(0.6,0.4),(0.5,0)\} \\
& =\left\{\frac{0.9}{0.8}, \frac{0.5}{0.7}, \frac{0.4}{0.6}\right\}
\end{aligned}
$$

For $\mathrm{p}=4, \mu_{Q}(4)=\left\{\left(u_{i}, \mu_{u i}(4)\right): i=1,2,3,4\right\}=$ $\{(0.8,1),(0.7,0),(0.6,0),(0.5,0)\}=\left\{\frac{1}{0.8}\right\}$

For $p=5, \mu_{Q}(5)=\left\{\left(u_{i}, \mu_{u i}(5)\right): i=1,2,3,4\right\}=$ $\{(0.8,0.8),(0.7,0.4),(0.6,0.3),(0.5,0.2)\}=\left\{\frac{0.8}{0.8}, \frac{0.4}{0.7}\right.$, $\left.\frac{0.3}{0.6}, \frac{0.2}{0.5}\right\}$ For $\mathrm{p}=6, \mu_{Q}(6)=\left\{\left(u_{i}, \mu_{u i}(6)\right): i=1,2,3\right\}=$ $\{(0.8,0),(0.7,0),(0.6,0.5),(0.5,0.3)\}=\left\{\frac{0.5}{0.6}, \frac{0.3}{0.5}\right\}$

Therefore, the type-2 fuzzy set

$$
\begin{aligned}
Q= & \left\{\frac{\left\{\frac{0.9}{0.8}, \frac{0.5}{0.7}, \frac{0.4}{0.6}\right\}}{3},\right. \\
& \frac{\left\{\frac{1}{0.8}\right\}}{4}, \frac{\left\{\frac{0.8}{0.8}, \frac{0.4}{0.7}, \frac{0.3}{0.6}, \frac{0.2}{0.5}\right\}}{5}, \\
& \left.\frac{\left\{\frac{0.5}{0.6}, \frac{0.3}{0.5}\right\}}{6}\right\}
\end{aligned}
$$

Example 6 Let $X=\{1,2,3,4\}$. Suppose $\left\{u_{j}: u_{j} \in[0,1]\right\}=$ $\{0,0.2,0.4,0.6\}$ be the primary membership, that is, the domain of the secondary membership $\mu_{A}(x)$ which also depicted in Fig. 5. The type- 2 membership function $\mu_{A}(x)$ in Fig. 5 has four vertical slices connected with it. Then sec- ondary membership function at $x=2$ is given by

$$
\begin{aligned}
\mu_{A}(2) & =\left\{\left(u_{j}, \mu_{u j}(2)\right): j=1,2,3,4\right\} \\
& =\{(0,1),(0.2,0.5),(0.4, .3),(0.6,0.3)\} \\
& =\left\{\frac{1}{0}, \frac{0.5}{0.2}, \frac{0.3}{0.4}, \frac{0.3}{0.6}\right\}
\end{aligned}
$$

Similarly for $x=1,3,4$ are respectively,

$$
\begin{aligned}
\mu_{A}(1) & =\left\{\left(u_{j}, \mu_{u j}(1)\right): j=1,2,3,4\right\} \\
& =\{(0,1),(0.2,0.8),(0.4,0.9),(0.6,0.2)\} \\
& =\left\{\frac{1}{0}, \frac{0.8}{0.2}, \frac{0.9}{0.4}, \frac{0.2}{0.6}\right\} \\
\mu_{A}(3) & =\left\{\left(u_{j}, \mu_{u j}(3)\right): j=1,2,3,4\right\} \\
& =\{(0,0),(0.2,0),(0.4,0),(0.6,0.2)\} \\
& =\left\{\frac{0.2}{0.6}\right\} \\
\mu_{A}(4) & =\left\{\left(u_{j}, \mu_{u j}(4)\right): j=1,2,3,4\right\} \\
& =\{(0,0.3),(0.2,0.6),(0.4,0.4),(0.6,0.2)\} \\
& =\left\{\frac{0.3}{0}, \frac{0.6}{0.2}, \frac{0.4}{0.4}, \frac{0.2}{0.6}\right\}
\end{aligned}
$$

Therefore the type-2 fuzzy-set A is given by

$$
\begin{aligned}
\mathrm{A}= & \left\{\frac{\left\{\frac{1}{0}, \frac{0.8}{0.2}, \frac{0.9}{0.4}, \frac{0.2}{0.6}\right\}}{1},\right. \\
& \frac{\left\{\frac{1}{0}, \frac{0.5}{0.2}, \frac{0.3}{0.4}, \frac{0.3}{0.6}\right\}}{2}, \frac{\left\{\frac{.2}{0.6}\right\}}{3}, \\
& \left.\frac{\left\{\frac{0.3}{0}, \frac{0.6}{0.2}, \frac{0.4}{0.4}, \frac{0.2}{0.6}\right\}}{4}\right\}
\end{aligned}
$$

\section{Representation of a T2FSS}

If $J_{p}(\subseteq I=[0,1])$ be discrete in Definition 3, then the e-approximate elements of a T2FSS (F,Q), can be written as,

$$
\begin{aligned}
F(e) & =\left\{\left(p, \mu_{F(e)}(p)\right): p \in U\right\} \\
& =\left\{\left(p,\left(u_{i}, \mu_{u i}(p)\right)\right): p \in U ; u_{i}, \mu_{u i}(p) \in[0,1]\right\} \\
& =\left\{\frac{\left(u_{i}, \mu_{u i}\right)}{p}: p \in U ; u_{i}, \mu_{u i} \in[0,1]\right\}
\end{aligned}
$$

Here $\mathrm{U}$ be the initial universe and $\mu_{F(e)}(p)$ is the secondary membership of the type-2 fuzzy-set $F(e)$ in $\mathrm{U}$. The domain of secondary membership $\mu_{F(e)}(p): u_{i} \rightarrow \mu_{u i}(p)$ is $\left\{u_{i}\right.$ : $\left.u_{i} \in[0,1]\right\}$ which is the primary membership of $F(e)$ in $\mathrm{U}$. 
Fig. 5 Type-2 membership function

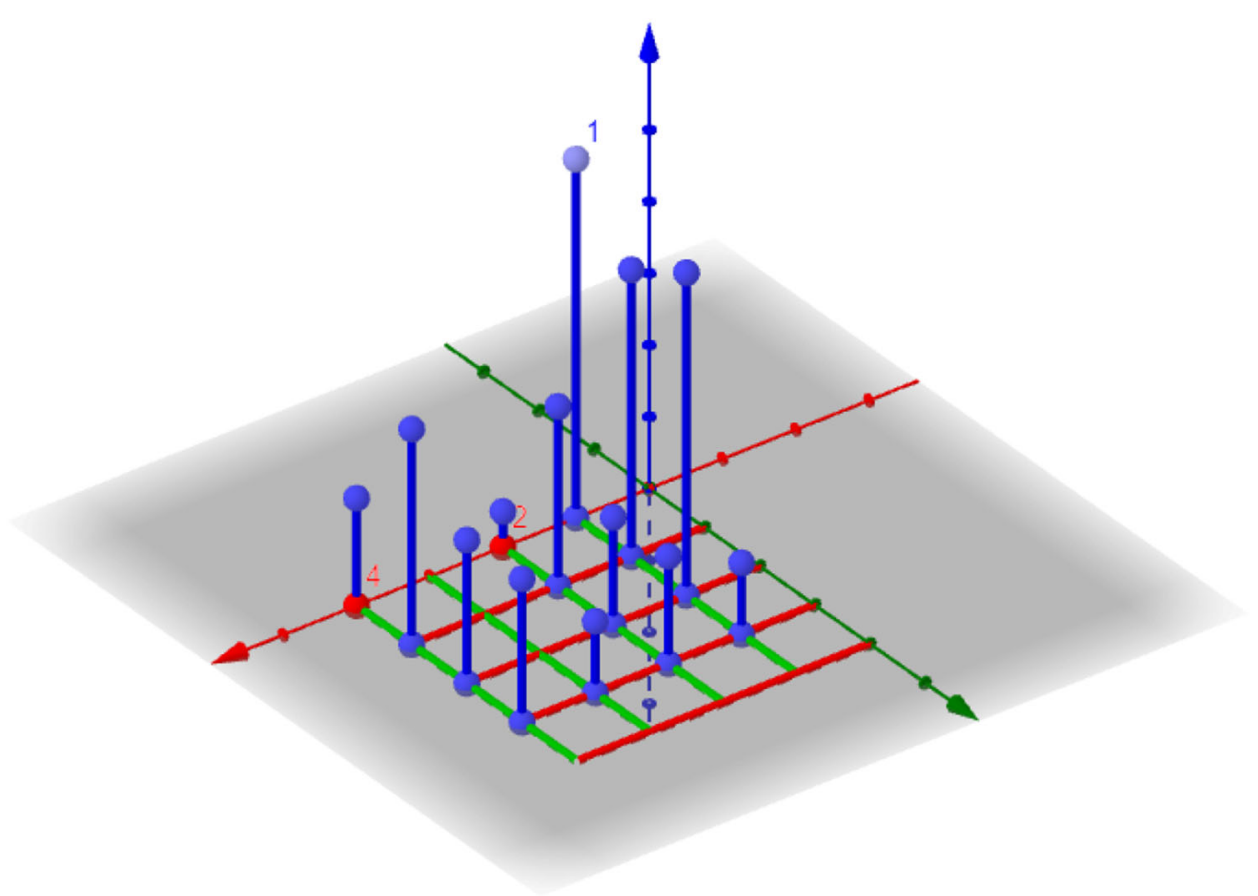

Example 7 Suppose, a $\operatorname{T} 2 \mathrm{FSS}(\mathcal{F}, Q)$ is

$$
\begin{aligned}
& (\mathcal{F}, Q)=\left\{b_{1}\right. \\
& =\left\{\frac{\left\{\frac{0.3}{0.1}, \frac{1.0}{0.2}, \frac{0.7}{0.3}\right\}}{a_{1}}, \frac{\left\{\frac{0.4}{0.3}, \frac{1.0}{0.5}, \frac{0.3}{0.6}\right\}}{a_{2}},\right. \\
& \frac{\left\{\frac{0.6}{0.5}, \frac{1.0}{0.9}\right\}}{a_{3}}, \frac{\left\{\frac{0.3}{0.4}, \frac{0.8}{0.5}, \frac{0.2}{0.6}\right\}}{a_{4}}, \frac{\left\{\frac{0.5}{0.7}\right\}}{a_{5}}, \\
& \left.\frac{\left\{\frac{0.3}{0.1}, \frac{1.0}{0.6}, \frac{0.5}{0.7}, \frac{0.2}{0.9}\right\}}{a_{6}}\right\}, \\
& b_{2}=\left\{\frac{\left\{\frac{0.3}{0.1}, \frac{0.6}{0.4}, \frac{0.5}{0.7}, \frac{0.5}{0.8}\right\}}{a_{1}},\right. \\
& \frac{\left\{\frac{0.1}{0.1}, \frac{0.6}{0.2}, \frac{0.9}{0.6}\right\}}{a_{2}}, \frac{\left\{\frac{0.7}{0.8}\right\}}{a_{3}}, \frac{\left\{\frac{0.9}{0.4}\right\}}{a_{4}}, \frac{\left\{\frac{0.6}{0.5}, \frac{1.0}{0.6}\right\}}{a_{5}}, \\
& \left.\frac{\left\{\frac{0.6}{0.1}, \frac{0.7}{0.4}\right\}}{a_{6}}\right\} \\
& b_{3}=\left\{\frac{\left\{\frac{0.2}{0.6}, \frac{0.8}{0.8}, \frac{0.6}{0.9}\right\}}{a_{1}}, \frac{\left\{\frac{0.6}{0.4}, \frac{0.8}{0.7}\right\}}{a_{2}},\right. \\
& \frac{\left\{\frac{0.5}{0.3}, \frac{0.9}{0.4}, \frac{0.4}{0.6}\right\}}{a_{3}}, \frac{\left\{\frac{0.3}{0.5}, \frac{0.9}{0.6}, \frac{0.4}{0.8}\right\}}{a_{4}}, \frac{\left\{\frac{0.6}{0.5}, \frac{1.0}{0.6}\right\}}{a_{5}},
\end{aligned}
$$

$$
\begin{aligned}
& \left.\frac{\left\{\frac{1.0}{0.9}\right\}}{a_{6}}\right\} \\
& b_{4}=\left\{\frac{\left\{\frac{0.5}{0.7}\right\}}{a_{1}}, \frac{\left\{\frac{0.3}{0.1}, \frac{0.8}{0.5}, \frac{0.5}{0.8}\right\}}{a_{2}}, \frac{\left\{\frac{0.7}{0.4}, \frac{0.7}{0.8}\right\}}{a_{3}},\right. \\
& \frac{\left\{\frac{0.2}{0.4}, \frac{0.7}{0.5}, \frac{0.5}{0.6}\right\}}{a_{4}}, \frac{\left\{\frac{0.7}{0.1}, \frac{0.6}{0.2}, \frac{0.3}{0.5}, \frac{0.5}{0.6}\right\}}{a_{5}}, \\
& \left.\frac{\left\{\frac{0.4}{0.5}, \frac{0.3}{0.7}\right\}}{a_{6}}\right\} \\
& b_{5}=\left\{\frac{\left\{\frac{0.9}{0.5}, \frac{1.0}{0.8}\right\}}{a_{1}}, \frac{\left\{\frac{0.1}{0.1}, \frac{0.9}{0.5}, \frac{0.6}{0.6}\right\}}{a_{2}},\right. \\
& \frac{\left\{\frac{0.3}{0.2}, \frac{0.7}{0.4}, \frac{0.8}{0.5}, \frac{0.9}{0.7}\right\}}{a_{3}}, \frac{\left\{\frac{0.7}{0.5}, \frac{1.0}{0.8}\right\}}{a_{4}}, \frac{\left\{\frac{0.9}{0.5}\right\}}{a_{5}}, \\
& \left.\left.\frac{\left\{\frac{0.2}{0.6}, \frac{0.5}{0.7}, \frac{0.4}{0.9}\right\}}{a_{6}}\right\}\right\}
\end{aligned}
$$

Here the $b_{1}$-approximate, $b_{2}$-approximate, $b_{3}$-approximate, $b_{4}$-approximate and $b_{5}$-approximate elements of the T2FSS $(\mathcal{F}, Q)$ as,

$F\left(b_{1}\right)=\left\{\frac{\left\{\frac{0.3}{0.1}, \frac{1.0}{0.2}, \frac{0.7}{0.3}\right\}}{a_{1}}\right.$, 


$$
\begin{aligned}
& \frac{\left\{\frac{0.4}{0.3}, \frac{1.0}{0.5}, \frac{0.3}{0.6}\right\}}{a_{2}}, \frac{\left\{\frac{0.6}{0.5}, \frac{1.0}{0.9}\right\}}{a_{3}}, \\
& \left.\frac{\left\{\frac{0.3}{0.4}, \frac{0.8}{0.5}, \frac{0.2}{0.6}\right\}}{a_{4}}, \frac{\left\{\frac{0.5}{0.7}\right\}}{a_{5}}, \frac{\left\{\frac{0.3}{0.1}, \frac{1.0}{0.6}, \frac{0.5}{0.7}, \frac{0.2}{0.9}\right\}}{a_{6}}\right\} \\
& F\left(b_{2}\right)=\left\{\frac{\left\{\frac{0.3}{0.1}, \frac{0.6}{0.4}, \frac{0.5}{0.7}, \frac{0.5}{0.8}\right\}}{a_{1}}, \frac{\left\{\frac{0.1}{0.1}, \frac{0.6}{0.2}, \frac{0.9}{0.6}\right\}}{a_{2}},\right. \\
& \frac{\left\{\frac{0.7}{0.8}\right\}}{a_{3}}, \frac{\left\{\frac{0.9}{0.4}\right\}}{a_{4}}, \\
& \left.\frac{\left\{\frac{0.6}{0.5}, \frac{1.0}{0.6}\right\}}{a_{5}}, \frac{\left\{\frac{0.6}{0.1}, \frac{0.7}{0.4}\right\}}{a_{6}}\right\} \text {, } \\
& F\left(b_{3}\right)=\left\{\frac{\left\{\frac{0.2}{0.6}, \frac{0.8}{0.8}, \frac{0.6}{0.9}\right\}}{a_{1}}, \frac{\left\{\frac{0.6}{0.4}, \frac{0.8}{0.7}\right\}}{a_{2}}, \frac{\left\{\frac{0.5}{0.3}, \frac{0.9}{0.4}, \frac{0.4}{0.6}\right\}}{a_{3}},\right. \\
& \left.\frac{\left\{\frac{0.3}{0.5}, \frac{0.9}{0.6}, \frac{0.4}{0.8}\right\}}{a_{4}}, \frac{\left\{\frac{0.6}{0.5}, \frac{1.0}{0.6}\right\}}{a_{5}}, \frac{\left\{\frac{1.0}{0.9}\right\}}{a_{6}}\right\}, \\
& F\left(b_{4}\right)=\left\{\frac{\left\{\frac{0.5}{0.7}\right\}}{a_{1}}, \frac{\left\{\frac{0.3}{0.1}, \frac{0.8}{0.5}, \frac{0.5}{0.8}\right\}}{a_{2}}, \frac{\left\{\frac{0.7}{0.4}, \frac{0.7}{0.8}\right\}}{a_{3}},\right. \\
& \frac{\left\{\frac{0.2}{0.4}, \frac{0.7}{0.5}, \frac{0.5}{0.6}\right\}}{a_{4}}, \\
& \left.\frac{\left\{\frac{0.7}{0.1}, \frac{0.6}{0.2}, \frac{0.3}{0.5}, \frac{0.5}{0.6}\right\}}{a_{5}}, \frac{\left\{\frac{0.4}{0.5}, \frac{0.3}{0.7}\right\}}{a_{6}}\right\}, \\
& F\left(b_{5}\right)=\left\{\frac{\left\{\frac{0.9}{0.5}, \frac{1.0}{0.8}\right\}}{a_{1}}, \frac{\left\{\frac{0.1}{0.1}, \frac{0.9}{0.5}, \frac{0.6}{0.6}\right\}}{a_{2}},\right. \\
& \frac{\left\{\frac{0.3}{0.2}, \frac{0.7}{0.4}, \frac{0.8}{0.5}, \frac{0.9}{0.7}\right\}}{a_{3}} \\
& \left.\frac{\left\{\frac{0.7}{0.5}, \frac{1.0}{0.8}\right\}}{a_{4}}, \frac{\left\{\frac{0.9}{0.5}\right\}}{a_{5}}, \frac{\left\{\frac{0.2}{0.6}, \frac{0.5}{0.7}, \frac{0.4}{0.9}\right\}}{a_{6}}\right\} \\
& \frac{\left\{\frac{0.6}{0.2}, \frac{0.1}{0.4}\right\}}{a_{3}}, \frac{\left\{\frac{0.3}{0.2}, \frac{0.1}{0.4}, \frac{0.2}{0.8}\right\}}{a_{4}}, \frac{\left\{\frac{0.1}{0.8}\right\}}{a_{5}}, \\
& \left.\frac{\left\{\frac{0.3}{0.2}, \frac{0.1}{0.4}, \frac{0.5}{0.8}\right\}}{a_{6}}\right\} \text {, } \\
& b_{2}=\left\{\frac{\left\{\frac{0.3}{0.1}, \frac{0.1}{0.4}, \frac{0.5}{0.8}\right\}}{a_{1}},\right. \\
& \frac{\left\{\frac{0.1}{0.1}, \frac{0.6}{0.4}, \frac{0.1}{0.8}\right\}}{a_{2}}, \frac{\left\{\frac{0.1}{0.1}\right\}}{a_{3}}, \frac{\left\{\frac{0.1}{0.1}\right\}}{a_{4}}, \\
& \left.\frac{\left\{\frac{0.6}{0.1}, \frac{0.1}{0.8}\right\}}{a_{5}}, \frac{\left\{\frac{0.6}{0.1}, \frac{0.1}{0.8}\right\}}{a_{6}}\right\} \text {, } \\
& b_{3}=\left\{\frac{\left\{\frac{0.2}{0.2}, \frac{0.1}{0.4}, \frac{0.6}{0.9}\right\}}{a_{1}}, \frac{\left\{\frac{0.6}{0.4}, \frac{0.1}{0.9}\right\}}{a_{2}},\right. \\
& \frac{\left\{\frac{0.5}{0.2}, \frac{0.1}{0.4}, \frac{0.4}{0.9}\right\}}{a_{3}}, \frac{\left\{\frac{0.3}{0.2}, \frac{0.1}{0.4}, \frac{0.4}{0.9}\right\}}{a_{4}}, \\
& \left.\frac{\left\{\frac{0.6}{0.4}, \frac{1.0}{0.9}\right\}}{a_{5}}, \frac{\left\{\frac{0.1}{0.9}\right\}}{a_{6}}\right\} \text {, } \\
& b_{4}=\left\{\frac{\left\{\frac{0.1}{0.5}\right\}}{a_{1}}, \frac{\left\{\frac{0.3}{0.4}, \frac{0.1}{0.5}, \frac{0.5}{0.8}\right\}}{a_{2}}, \frac{\left\{\frac{0.7}{0.5}, \frac{0.1}{0.8}\right\}}{a_{3}},\right. \\
& \left.\frac{\left\{\frac{0.2}{0.4}, \frac{0.1}{0.5}, \frac{0.5}{0.8}\right\}}{a_{4}}, \frac{\left\{\frac{0.1}{0.4}, \frac{0.6}{0.5}, \frac{0.3}{0.8}\right\}}{a_{5}}, \frac{\left\{\frac{0.1}{0.4}, \frac{0.3}{0.5}\right\}}{a_{6}}\right\}, \\
& b_{5}=\left\{\frac{\left\{\frac{0.9}{0.2}, \frac{0.1}{0.5}\right\}}{a_{1}}, \frac{\left\{\frac{0.1}{0.2}, \frac{0.1}{0.4}, \frac{0.6}{0.5}\right\}}{a_{2}},\right. \\
& \frac{\left\{\frac{0.3}{0.2}, \frac{0.7}{0.4}, \frac{0.1}{0.5}\right\}}{a_{3}}, \frac{\left\{\frac{0.7}{0.4}, \frac{0.1}{0.5}\right\}}{a_{4}}, \\
& \left.\left.\frac{\left\{\frac{0.1}{0.5}\right\}}{a_{5}}, \frac{\left\{\frac{0.2}{0.2}, \frac{0.1}{0.4}, \frac{0.4}{0.5}\right\}}{a_{6}}\right\}\right\}
\end{aligned}
$$

respectively.

Table 1 shows the tabular representation of T2FSS $(\mathcal{F}, Q)$

Example 8 Suppose, a T2FSS $(\mathcal{F}, Q)$ is

$(\mathcal{F}, Q)=\left\{b_{1}=\left\{\frac{\left\{\frac{0.3}{0.2}, \frac{0.1}{0.4}, \frac{0.8}{0.8}\right\}}{a_{1}}, \frac{\left\{\frac{0.2}{0.2}, \frac{0.1}{0.4}, \frac{0.3}{0.8}\right\}}{a_{2}}, \quad F\left(b_{1}\right)=\left\{b_{1}=\left\{\frac{\left\{\frac{0.3}{0.2}, \frac{0.1}{0.4}, \frac{0.8}{0.8}\right\}}{a_{1}}\right.\right.\right.\right.$,
Here $U=\left\{a_{1}, a_{2}, a_{3}, a_{4}, a_{5}, a_{6}\right\}$ and $\left\{u_{j}: u_{j} \in[0,1]\right\}=$ $\{0.2,0.4,0.8\}$ be the primary membership, that is, the domain of the secondary membership $\mu_{F(e)}(p)$ in $F\left(b_{1}\right)$. Then the $b_{1}$-approximate element of the $\operatorname{T} 2 \mathrm{FSS}(\mathcal{F}, Q)$ is, 
Table 1 T2FSS $(\mathcal{F}, Q)$ in tabular representation

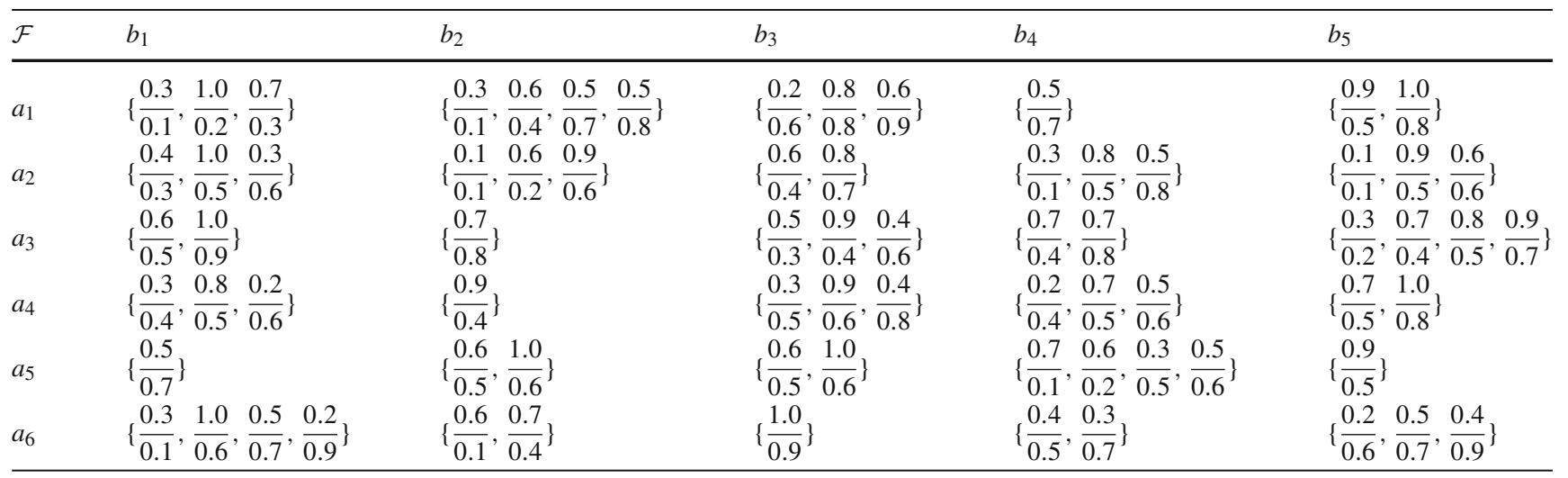

$$
\begin{aligned}
& \frac{\left\{\frac{0.2}{0.2}, \frac{0.1}{0.4}, \frac{0.3}{0.8}\right\}}{a_{2}}, \frac{\left\{\frac{0.6}{0.2}, \frac{0.1}{0.4}\right\}}{a_{3}}, \\
& \left\{\frac{0.3}{0.2}, \frac{0.1}{0.4}, \frac{0.2}{0.8}\right\} \\
& \left\{\frac{0.1}{a_{4}}\right\} \\
& \left.\frac{a_{5}}{0.8}, \frac{\left\{\frac{0.3}{0.2}, \frac{0.1}{0.4}, \frac{0.5}{0.8}\right\}}{a_{6}}\right\},
\end{aligned}
$$

Similarly the $b_{2}$-approximate, $b_{3}$-approxim ate, $b_{4}$-approximate and $b_{5}$-approximate elements of the T2FSS $(\mathcal{F}, Q)$ as,

$$
\begin{aligned}
& F\left(b_{2}\right)=\left\{\frac{\left\{\frac{0.3}{0.1}, \frac{0.1}{0.4}, \frac{0.5}{0.8}\right\}}{a_{1}}, \frac{\left\{\frac{0.1}{0.1}, \frac{0.6}{0.4}, \frac{0.1}{0.8}\right\}}{a_{2}}, \frac{\left\{\frac{0.1}{0.1}\right\}}{a_{3}},\right. \\
& \left.\frac{\left\{\frac{0.1}{0.1}\right\}}{a_{4}}, \frac{\left\{\frac{0.6}{0.1}, \frac{0.1}{0.8}\right\}}{a_{5}}, \frac{\left\{\frac{0.6}{0.1}, \frac{0.1}{0.8}\right\}}{a_{6}}\right\} \text {, } \\
& F\left(b_{3}\right)=\left\{\frac{\left\{\frac{0.2}{0.2}, \frac{0.1}{0.4}, \frac{0.6}{0.9}\right\}}{a_{1}}, \frac{\left\{\frac{0.6}{0.4}, \frac{0.1}{0.9}\right\}}{a_{2}},\right. \\
& \frac{\left\{\frac{0.5}{0.2}, \frac{0.1}{0.4}, \frac{0.4}{0.9}\right\}}{a_{3}}, \\
& \left.\frac{\left\{\frac{0.3}{0.2}, \frac{0.1}{0.4}, \frac{0.4}{0.9}\right\}}{a_{4}}, \frac{\left\{\frac{0.6}{0.4}, \frac{1.0}{0.9}\right\}}{a_{5}}, \frac{\left\{\frac{0.1}{0.9}\right\}}{a_{6}}\right\}, \\
& F\left(b_{4}\right)=\left\{\frac{\left\{\frac{0.1}{0.5}\right\}}{a_{1}}, \frac{\left\{\frac{0.3}{0.4}, \frac{0.1}{0.5}, \frac{0.5}{0.8}\right\}}{a_{2}}, \frac{\left\{\frac{0.7}{0.5}, \frac{0.1}{0.8}\right\}}{a_{3}},\right. \\
& \left.\frac{\left\{\frac{0.2}{0.4}, \frac{0.1}{0.5}, \frac{0.5}{0.8}\right\}}{a_{4}}, \frac{\left\{\frac{0.1}{0.4}, \frac{0.6}{0.5}, \frac{0.3}{0.8}\right\}}{a_{5}}, \frac{\left\{\frac{0.1}{0.4}, \frac{0.3}{0.5}\right\}}{a_{6}}\right\},
\end{aligned}
$$

respectively.

\section{Motivation}

\section{Zhang et al. [39]}

Zhang et al. [39] introduced T2FSS by combining the type- 2 fuzzy-set theory with soft-set theory. Type-2 fuzzy-soft-set theory successfully deals with complicated problems with uncertainty, and its representation is more complicated than the classical fuzzy-soft-set theory. In the previous section, we have already represented the T2FSS in its simplest form and visually understand some pictorial examples. Zhang et al. [39] also suggested a method to solve T2FSS based DMP. They developed an algorithm by collecting two inputs from a decision-maker, namely, $\alpha$ threshold and $\lambda$ threshold. But in the practical situation, a decision-maker may face a problem to provide the information as those inputs are in a fuzzy concept. By the above motivation, here we have defined the mid $\alpha$-threshold fuzzy-set (Definition 6) and the mid $\lambda$-threshold fuzzy-set (Definition 7) of a type-2 fuzzy soft set.

Further, with the help of these two definitions, we have proposed two algorithms by collecting only the choice parameters (and their weights if necessary) from the decisionmaker. Finally, we are going to solve three real-life problems using these two algorithms. We also claim that this is an 
Table 2 Level soft set $((\mathcal{F}, Q) ; \alpha, \lambda)$ in tabular form with choice values

\begin{tabular}{lllllll}
\hline $\mathcal{F}$ & $b_{1}$ & $b_{2}$ & $b_{3}$ & $b_{4}$ & $b_{5}$ & Choice values $u_{i}$ \\
\hline$a_{1}$ & 0 & 1 & 1 & 1 & 0 & $v_{1}=3$ \\
$a_{2}$ & 1 & 0 & 0 & 1 & 0 & $v_{2}=2$ \\
$a_{3}$ & 1 & 1 & 0 & 0 & 0 & $v_{3}=2$ \\
$a_{4}$ & 1 & 1 & 1 & 1 & 0 & $v_{4}=4$ \\
$a_{5}$ & 0 & 1 & 0 & 0 & 0 & $v_{5}=1$ \\
$a_{6}$ & 1 & 0 & 1 & 0 & 0 & $v_{6}=2$ \\
\hline
\end{tabular}

important method to solve a T2FSS based DMP. Z. Zhang's process takes more input values from the decision-maker to develop their algorithm. But these input values are all in the fuzzy sense. So the input values vary according to different decision-makers. Hence the decision-making result may differ according to other decision-makers' opinions. Our motivation in this paper is to minimize the number of input values and consistently develop a suitable algorithm.

Zhang et al. [39] proposed a definition, called, Levelsoft-set of a type-2 fuzzy-soft-set (Definition 4). Let us try to understand this definition by considering the Example 7 with $\alpha=\left\{\frac{0.6}{b_{1}}, \frac{0.6}{b_{2}}, \frac{0.6}{b_{3}}, \frac{0.5}{b_{4}}, \frac{0.7}{b_{5}}\right\}$ and $\lambda=$ $\left\{\frac{0.5}{b_{1}}, \frac{0.4}{b_{2}}, \frac{0.6}{b_{3}}, \frac{0.5}{b_{4}}, \frac{0.6}{b_{5}}\right\}$. Then we have the following results : $\mathcal{F}\left(b_{1}\right)_{0.5}^{0.6}=\left\{a_{2}, a_{3}, a_{4}, a_{6}\right\}, \mathcal{F}\left(b_{2}\right)_{0.4}^{0.6}=\left\{a_{1}, a_{3}, a_{4}\right.$, $\left.a_{5}\right\}, \mathcal{F}\left(b_{3}\right)_{0.6}^{0.6}=\left\{a_{1}, a_{4}, a_{6}\right\}, \mathcal{F}\left(b_{4}\right)_{0.5}^{0.5}=\left\{a_{1}, a_{2}, a_{4}\right\}$, $\mathcal{F}\left(b_{5}\right)_{0.6}^{0.7}=\{\}=\phi$. Table 2 gives the representation in tabular form of $(\alpha, \lambda)$-level fuzzy set $L((\mathcal{F}, Q) ; \alpha, \lambda))$ with choice values.

Let us try to analyze the portion " $\mathcal{F}\left(b_{1}\right)_{0.5}^{0.6}=\left\{a_{2}, a_{3}, a_{4}\right.$, $\left.a_{6}\right\}^{\prime \prime}$. Here, for $b_{1}, J_{a_{1}}^{0.6}=\left\{u: \mu_{F\left(e_{1}\right)}(x, u) \geq 0.6, u \in\right.$ $\left.J_{a_{1}}\right\}=\{0.2,0.3\}$. Similarly, $J_{a_{2}}^{0.6}=\{0.5\}, J_{a_{3}}^{0.6}=\{0.5,0.9\}$, $J_{a_{4}}^{0.6}=\{0.5\}, J_{a_{5}}^{0.6}=\{\}$ and $J_{a_{6}}^{0.6}=\{0.6\}$. Therefore, $\mathcal{F}\left(b_{1}\right)_{0.5}^{0.6}=\left\{x \in U: u(x) \geq 0.5, \forall u(x) \in J_{x}^{0.6}\right\}=$ $\left\{a_{2}, a_{3}, a_{4}, a_{5}\right\}$. Clearly, $a_{2}$ fulfill the condition " $\{u(x) \geq$ $\left.\lambda(e), \forall u(x) \in J_{x}^{0.6}\right\}^{\prime \prime}$ by single value, namely, o.5; where as $a_{3}$ fulfill this condition by two values, namely, 0.5 and 0.9 . But, according to definition of level soft set (Definition 4), $a_{2}$ and $a_{3}$ has the equal weights (importance). To improve this situation, we are going to present, the definition 8 in Sect. 5 .

\section{Feng et al. [40]}

In decision making problems involving fuzzy soft sets, the situation becomes somewhat different and much more complicated. In particular, it is worth noting that the so-called choice value considered in [41] should be exactly interpreted as the "fuzzy choice value", which is just the sum total of all membership values with respect to different attributes. Thus for a fuzzy soft set, this choice value no longer represents the number of (good) attributes possessed by that object. On the other hand, it can be seen as a synthesized measure to estimate each object via a fusion of all attributes. However, this direct addition of all the membership values with respect to different attributes is not always reasonable. Consider, for instance, the set of round and coarse objects. While it may be reasonable to compare the roundness of two objects, it is not equally reasonable to add the degree of roundness of an object to the degree to which that object is coarse; in a certain sense this is just like, for instance, the addition of height and weight. To solve this difficulty associated with fuzzy soft sets, rather than using the concept of choice values designed for crisp soft sets, Roy and Maji [42] introduced the concept of a comparison table and a measure called the score of an object. Like most of the decision making problems, fuzzy soft set based decision making involves the evaluation of all the objects which are decision alternatives. Some of these problems are essentially humanistic and thus subjective in nature (e.g. human understanding and vision systems). In general, there does not exist a unique or uniform criterion for the evaluation of decision alternatives. In fact, the divergence of opinions in $[41,42]$ is whether scores or choice values should be used to estimate decision alternatives and to determine the optimal objects. Feng et al. [40] introduced an adjustable approach to fuzzy soft set based decision making problems to overcome the above difficulties. This proposal is based on the following novel concept called level soft sets.

Definition 5 Let $(F, P)$ be an FSS over a finite universal set $\mathrm{V}$, where $P \subseteq Q$ and $\mathrm{Q}$ is the set of all parameters. Let $\lambda: P \rightarrow[0,1]$ be a function(membership) on $\mathrm{P}$, the FS in $\mathrm{P}$, denoted by, $P_{\lambda}$ is called a threshold fuzzy set. A level soft set of the FS $(\mathrm{F}, \mathrm{P})$ w.r. to the FS $A_{\lambda}$ is a crisp soft-set $\left(F_{\lambda}, P\right)$ defined by $F_{\lambda}(a)=\{v \in V: F(a)(v) \geq \lambda(a), \forall a \in P\}$.

Inspired by these two papers, in the next section, we have proposed a definition, named as level-fuzzy-soft set(LFSS) of a T2FSS.

\section{Proposed definitions}

Definition 6 Mid- $\alpha$-threshold fuzzy-set of a T2FSS :

Let $(\mathcal{F}, P)$ be a $\mathrm{T} 2 \mathrm{FSS}$ over $\mathrm{V}$, where $\mathrm{P} \subseteq \mathrm{Q}$ and $\mathrm{Q}$ is the set of all parameters. Let $\alpha: P \rightarrow[0,1]$ be an FS in $\mathrm{P}$, called the mid- $\alpha$-threshold fuzzy set of a T2FSS $(\mathcal{F}, P)$, denoted by $\alpha=\left(b, \mu_{\alpha(b)}\right)$ where $\mu_{\alpha(b)}$ is an average crisp value of the degrees of secondary membership of $(\mathcal{F}, P)$ corresponding to $b \in P$.

Example 9 Let us consider the same T2FSS given in Example 7. Then by Definition 6,

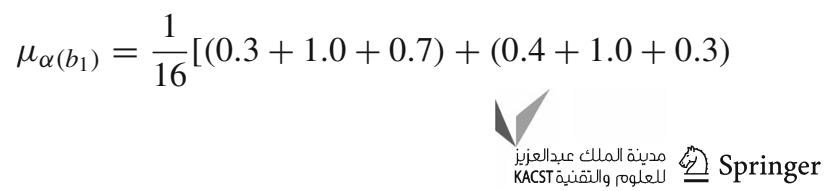

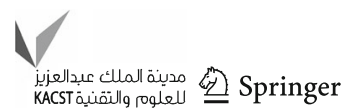




$$
\begin{aligned}
& +(0.6+1.0)+(0.3+0.8+0.2)+0.5 \\
& +(0.3+1.0+0.5+0.2)]=0.6
\end{aligned}
$$

Similarly, $\mu_{\alpha\left(b_{2}\right)}=0.6, \mu_{\alpha\left(b_{3}\right)}=0.6, \mu_{\alpha\left(b_{4}\right)}=0.5$, $\mu_{\alpha\left(b_{5}\right)}=0.7$

Therefore, $\alpha=\left\{\frac{0.6}{b_{1}}, \frac{0.6}{b_{2}}, \frac{0.6}{b_{3}}, \frac{0.5}{b_{4}}, \frac{0.7}{b_{5}}\right\}$

Example 10 Let us consider the same T2FSS given in Example 8 . Then by Definition 6 ,

$$
\begin{aligned}
& \mu_{\alpha\left(b_{1}\right)} \\
& \begin{aligned}
= & \frac{1}{15}[(0.3+0.1+0.8)+(0.2+0.1+0.3) \\
& +(0.6+0.1)+(0.3+0.1+0.2) \\
& +0.1+(0.3+.1+0.5)]=0.3
\end{aligned}
\end{aligned}
$$

Similarly, $\mu_{\alpha\left(b_{2}\right)}=0.3, \mu_{\alpha\left(b_{3}\right)}=0.4, \mu_{\alpha\left(b_{4}\right)}=0.3$, $\mu_{\alpha\left(b_{5}\right)}=0.3$

Therefore, $\alpha=\left\{\frac{0.3}{b_{1}}, \frac{0.3}{b_{2}}, \frac{0.4}{b_{3}}, \frac{0.3}{b_{4}}, \frac{0.3}{b_{5}}\right\}$

Definition 7 Mid- $\lambda$-threshold fuzzy-set of a T2FSS :

Let $(\mathcal{F}, P)$ is a $\mathrm{T} 2 \mathrm{FSS}$ over $\mathrm{V}$, where $\mathrm{P} \subseteq \mathrm{Q}$ and $\mathrm{Q}$ is the set of all parameters. Let $\lambda: P \rightarrow[0,1]$ is a FS in P, called mid- $\lambda$-threshold fuzzy-set of a T2FSS $(\mathcal{F}, P)$, denoted by $\lambda=\left(b, \mu_{\lambda(b)}\right)$ where $\mu_{\lambda(b)}$ is an average crisp value of the degrees of primary membership of $(\mathcal{F}, P)$ corresponding to $b \in P$.

Example 11 Let us consider the same T2FSS given in Example 7. Then by Definition 7, we get, $\mu_{\lambda\left(b_{1}\right)}=0.5, \mu_{\lambda\left(b_{2}\right)}=$ $0.4, \mu_{\lambda\left(b_{3}\right)}=0.6, \mu_{\lambda\left(b_{4}\right)}=0.5, \mu_{\lambda\left(b_{5}\right)}=0.6$.

Therefore, $\lambda=\left\{\frac{0.5}{b_{1}}, \frac{0.4}{b_{2}}, \frac{0.6}{b_{3}}, \frac{0.5}{b_{4}}, \frac{0.6}{b_{5}}\right\}$

Example 12 Let us consider the same T2FSS given in Example 8 . Then by Definition 7, we get, $\mu_{\lambda\left(b_{1}\right)}=0.5, \mu_{\lambda\left(b_{2}\right)}=$ $0.4, \mu_{\lambda\left(b_{3}\right)}=0.6, \mu_{\lambda\left(b_{4}\right)}=0.6, \mu_{\lambda\left(b_{5}\right)}=0.4$.

Therefore, $\lambda=\left\{\frac{0.5}{b_{1}}, \frac{0.4}{b_{2}}, \frac{0.6}{b_{3}}, \frac{0.6}{b_{4}}, \frac{0.4}{b_{5}}\right\}$

Definition 8 Level fuzzy soft set(LFSS) of a T2FSS

Let $(\mathcal{F}, P)$ be a T2FSS over $\mathrm{U}$, where $P \subseteq Q$ and $\mathrm{Q}$ is the set of all parameters. Let $\alpha: P \rightarrow[0,1]$ and $\lambda: P \rightarrow[0,1]$ be two fuzzy sets in P. The level fuzzy soft set(LFSS) of $(\mathcal{F}, P)$ with respect to $\alpha$ and $\lambda$ is a $\operatorname{FSS} \operatorname{L}((\mathcal{F}, P) ; \alpha, \lambda))=$ $\left(\mathcal{F}_{(\alpha, \lambda)}, P\right)$ defined as: for any $e \in P, \mathcal{F}_{(\alpha, \lambda)}(b)=\mathcal{F}(b)_{\lambda}^{\alpha}=$ $\left\{\left(p, \frac{n(u)}{1+n(\mu)}\right): p \in U, u(p) \geq \lambda(b), \forall u(p) \in J_{p}^{\alpha}\right\}$; where $\mathrm{n}(\mu)$ and $\mathrm{n}(\mathrm{u})$ are the numbers of $\mu$ 's and u's satisfying $\left\{\mu: \mu_{F(b)}(p, u) \geq \alpha(b)\right\}$ and $\{u: u(p) \geq \lambda(b), \forall u(p) \in$ $\left.J_{p}^{\alpha}\right\}, J_{p}^{\alpha}=\left\{u: \mu_{F(b)}(p, u) \geq \alpha(b), u \in J_{p} \subseteq I=[0,1]\right\}$.

The LFSS of a T2FSS w.r. to mid- $\alpha$-threshold fuzzy set (Definition 6) and mid- $\lambda$-threshold fuzzy set (Definition 7) is called mid level fuzzy soft set of the T2FSS.
Table 3 The tabular representation of $(\alpha, \lambda)$-level fuzzy soft set $((\mathcal{F}, Q) ; \alpha, \lambda)$ with choice values

\begin{tabular}{lllllll}
\hline $\mathcal{F}$ & $b_{1}$ & $b_{2}$ & $b_{3}$ & $b_{4}$ & $b_{5}$ & Choice values $v_{i}$ \\
\hline$a_{1}$ & 0 & 0.5 & 0.67 & 0.5 & 0.33 & $v_{1}=2$ \\
$a_{2}$ & 0.5 & 0.33 & 0.33 & 0.67 & 0 & $v_{2}=1.83$ \\
$a_{3}$ & 0.67 & 0.5 & 0 & 0.33 & 0.25 & $v_{3}=1.75$ \\
$a_{4}$ & 0.5 & 0.5 & 0.5 & 0.67 & 0.33 & $v_{4}=2.5$ \\
$a_{5}$ & 0 & 0.67 & 0.33 & 0.25 & 0 & $v_{5}=1.25$ \\
$a_{6}$ & 0.5 & 0.33 & 0.5 & 0 & 0 & $v_{6}=1.33$ \\
\hline
\end{tabular}

Example 13 Let us consider the Example 7 with $\alpha=$ $\left\{\frac{0.6}{b_{1}}, \frac{0.6}{b_{2}}, \frac{0.6}{b_{3}}, \frac{0.5}{b_{4}}, \frac{0.7}{b_{5}}\right\}$ and $\lambda=\left\{\frac{0.5}{b_{1}}, \frac{0.4}{b_{2}}, \frac{0.6}{b_{3}}, \frac{0.5}{b_{4}}\right.$, $\left.\frac{0.6}{b_{5}}\right\}$. Then we have the following results:

$$
\begin{aligned}
& \mathcal{F}\left(b_{1}\right)_{0.5}^{0.6}=\left\{\left(a_{2}, 0.5\right),\left(a_{3}, 0.67\right),\left(a_{4}, 0.5\right),\left(a_{6}, 0.5\right)\right\}, \\
& \mathcal{F}\left(b_{2}\right)_{0.4}^{0.6}=\left\{\left(a_{1}, 0.5\right),\left(a_{2}, 0.33\right),\left(a_{3}, 0.5\right),\right.\left.\left(a_{4}, 0.5\right),\left(a_{5}, 0.67\right),\left(a_{6}, 0.33\right)\right\} \\
& \mathcal{F}\left(b_{3}\right)_{0.6}^{0.6}=\left\{\left(a_{1}, 0.67\right),\left(a_{2}, 0.33\right),\left(a_{4}, 0.5\right),\right.\left.\left(a_{5}, 0.33\right),\left(a_{6}, 0.5\right)\right\} \\
& \mathcal{F}\left(b_{4}\right)_{0.5}^{0.5}=\left\{\left(a_{1}, 0.5\right),\left(a_{2}, 0.67\right),\left(a_{3}, 0.33\right),\right. \\
& \\
&\left.\mathcal{F}\left(b_{5}\right)_{0.6}^{0.7}=\left\{\left(a_{1}, 0.67\right),\left(a_{5}, 0.25\right)\right\},\left(a_{3}, 0.25\right),\left(a_{4}, 0.33\right)\right\}
\end{aligned}
$$

Therefore, the $(\alpha, \lambda)$-level fuzzy soft set of $(\mathcal{F}, Q)$ is a crisp or type-1 fuzzy-soft-set $((\mathcal{F}, Q) ; \alpha, \lambda)=\left(\mathcal{F}_{(\alpha, \lambda)}, Q\right)$ where the fuzzy set-valued mapping $\mathcal{F}_{(\alpha, \lambda)}: Q \rightarrow F(U)$ is defined by $\mathcal{F}_{(\alpha, \lambda)}\left(b_{i}\right)=\mathcal{F}\left(b_{i}\right)_{\lambda}^{\alpha}$ for $\mathrm{j}=1,2,3,4,5$. Thus $(\alpha, \lambda)$-level fuzzy soft set is $((\mathcal{F}, Q) ; \alpha, \lambda)=\left\{b_{1}=\right.$ $\left\{\frac{0.5}{a_{2}}, \frac{0.67}{a_{3}}, \frac{0.5}{a_{4}}, \frac{0.5}{a_{6}}\right\}, b_{2}=\left\{\frac{0.5}{a_{1}}, \frac{0.33}{a_{2}}, \frac{0.5}{a_{3}}, \frac{0.5}{a_{4}}, \frac{0.67}{a_{5}}\right.$, $\left.\frac{0.33}{a_{6}}\right\}, b_{3}=\left\{\frac{0.67}{a_{1}}, \frac{0.33}{a_{2}}, \frac{0.5}{a_{4}}, \frac{0.33}{a_{5}}, \frac{0.5}{a_{6}}\right\}, b_{4}=\left\{\frac{0.5}{a_{1}}\right.$, $\left.\frac{0.67}{a_{2}}, \frac{0.33}{a_{3}}, \frac{0.67}{a_{4}}, \frac{0.25}{a_{5}}\right\}, b_{5}=\left\{\frac{0.33}{a_{1}}, \frac{0.25}{a_{3}}, \frac{0.33}{a_{4}}\right\}$.

Table 3 shows the representation in tabular form of $(\alpha, \lambda)$ level fuzzy soft set $L((\mathcal{F}, Q) ; \alpha, \lambda))$.

Example 14 Let us consider the Example 8 with $\alpha=$ $\left\{\frac{0.3}{b_{1}}, \frac{0.3}{b_{2}}, \frac{0.4}{b_{3}}, \frac{0.3}{b_{4}}, \frac{0.3}{b_{5}}\right\}$ and $\lambda=\left\{\frac{0.5}{b_{1}}, \frac{0.4}{b_{2}}, \frac{0.6}{b_{3}}, \frac{0.6}{b_{4}}\right.$, $\left.\frac{0.4}{b_{5}}\right\}$. Then we have the following results:

$\mathcal{F}\left(b_{1}\right)_{0.5}^{0.3}=\left\{\left(a_{1}, 0.33\right),\left(a_{2}, 0.5\right),\left(a_{6}, 0.33\right)\right\}$,

$\mathcal{F}\left(b_{2}\right)_{0.4}^{0.3}=\left\{\left(a_{1}, 0.67\right),\left(a_{2}, 0.5\right)\right\}$,

$\mathcal{F}\left(b_{3}\right)_{0.6}^{0.4}=\left\{\left(a_{1}, 0.5\right),\left(a_{2}, 0\right),\left(a_{3}, 0.33\right),\left(a_{4}, 0.5\right),\left(a_{5}, 0.33\right)\right\}$,

$\mathcal{F}\left(b_{4}\right)_{0.6}^{0.3}=\left\{\left(a_{2}, 0.33\right),\left(a_{4}, 0.5\right),\left(a_{5}, 0.33\right)\right\}$,

$\mathcal{F}\left(b_{5}\right)_{0.4}^{0.3}=\left\{\left(a_{2}, 0.5\right),\left(a_{3}, 0.33\right),\left(a_{4}, 0.5\right),\left(a_{6}, 0.5\right)\right\}$ 
Table 4 The tabular representation of $(\alpha, \lambda)$-level fuzzy soft set $((\mathcal{F}, Q) ; \alpha, \lambda)$ with choice values

\begin{tabular}{lllllll}
\hline $\mathcal{F}$ & $b_{1}$ & $b_{2}$ & $b_{3}$ & $b_{4}$ & $b_{5}$ & Choice values $v_{i}$ \\
\hline$a_{1}$ & 0.33 & 0.67 & 0.5 & 0 & 0 & $v_{1}=1.5$ \\
$a_{2}$ & 0.5 & 0.5 & 0 & 0.33 & 0.5 & $v_{2}=1.83$ \\
$a_{3}$ & 0 & 0 & 0.33 & 0 & 0.33 & $v_{3}=0.66$ \\
$a_{4}$ & 0 & 0 & 0.5 & 0.5 & 0.5 & $v_{4}=1.5$ \\
$a_{5}$ & 0 & 0 & 0.33 & 0.33 & 0 & $v_{5}=0.66$ \\
$a_{6}$ & 0.33 & 0 & 0 & 0 & 0.5 & $v_{6}=0.83$ \\
\hline
\end{tabular}

Therefore, the $(\alpha, \lambda)$-level fuzzy soft set of $(\mathcal{F}, Q)$ is a crisp or type-1 fuzzy-soft-set

$((\mathcal{F}, Q) ; \alpha, \lambda)=\left(\mathcal{F}_{(\alpha, \lambda)}, Q\right)$ where the fuzzy set-valued mapping $\mathcal{F}_{(\alpha, \lambda)}: Q \rightarrow F(U)$ is defined by $\mathcal{F}_{(\alpha, \lambda)}\left(b_{i}\right)=$ $\mathcal{F}\left(b_{i}\right)_{\lambda}^{\alpha}$ for $\mathrm{j}=1,2,3,4,5$. Thus $(\alpha, \lambda)$-level fuzzy soft set is $((\mathcal{F}, Q) ; \alpha, \lambda)=\left\{b_{1}=\left\{\frac{0.33}{a_{1}}, \frac{0.5}{a_{2}}, \frac{0.33}{a_{6}}\right\}, b_{2}=\right.$ $\left\{\frac{0.67}{a_{1}}, \frac{0.5}{a_{2}}\right\}, b_{3}=\left\{\frac{0.5}{a_{1}}, \frac{0.33}{a_{3}}, \frac{0.5}{a_{4}}, \frac{0.33}{a_{5}}\right\}, b_{4}=\left\{\frac{0.33}{a_{2}}\right.$, $\left.\frac{0.5}{a_{4}}, \frac{0.33}{a_{5}}\right\}, b_{5}=\left\{\frac{0.5}{a_{2}}, \frac{0.33}{a_{3}}, \frac{0.5}{a_{4}}, \frac{0.5}{a_{6}}\right\}$.

Table 4 shows the representation in tabular form of $(\alpha, \lambda)$ level fuzzy soft set $L((\mathcal{F}, Q) ; \alpha, \lambda))$.

\section{Proposed algorithm for T2FSS based DMP}

Step 1: Provide the T2FSS $(\mathcal{F}, Q)$.

Step 2: Find threshold fuzzy sets $\alpha$ as well as $\lambda$ according to the Definition 6 and Definition 7 respectively.

Step 3: Evaluate the $\operatorname{LFSS} L((\mathcal{F}, Q) ; \alpha, \lambda)$ of $(\mathcal{F}, Q)$ w.r. to the threshold fuzzy-sets $\alpha$ and $\lambda$.

Step 4: Compute tabular representation of the mid-level fuzzy-soft-set $L((\mathcal{F}, Q) ; \alpha, \lambda)$. For any $a_{i} \in V$, calculate the choice value $u_{i}$ for $a_{i}$.

Step 5: The optimal decision is to choose $a_{k}$ if $u_{k}=$ $\max _{a_{i} \in V}\left\{u_{i}\right\}$.

Step 6: If $\mathrm{k}$ has multiple values, any one of $a_{k}$ may be selected.

Now in the next section, we demonstrate the mathematical explanation of the above algorithm.

\section{Mathematical explanation}

Step 1: Let $(\mathcal{F}, Q)=\left\{b_{j}=\left\{\frac{\left\{\frac{u_{l}\left(a_{i}, b_{j}\right)}{\mu_{l}\left(a_{i}, b_{j}\right)}\right\}}{a_{i}}\right\}\right\}$.
Step 2: $\alpha=\left\{\frac{u_{j}}{b_{j}}\right\}$ and $\lambda=\left\{\frac{\mu_{j}}{b_{j}}\right\}$, where $u_{j}=\frac{1}{l} \sum u_{l}\left(a_{i}, b_{j}\right)$ and $\mu_{j}=\frac{1}{l} \sum \mu_{l}\left(a_{i}, b_{j}\right)$. Mid- $\alpha$-threshold fuzzy set can help to select the best alternatives with respect to the second membership values and mid$\lambda$-threshold fuzzy set can help to select the best alternative with respect to the primary membership values.

Step 3: $L((\mathcal{F}, Q) ; \alpha, \lambda)=\left\{b_{j}=\left\{\frac{d\left(a_{i}, b_{j}\right)}{a_{i}}\right\}\right\}$, where $d\left(a_{i}, b_{j}\right)=\frac{n(u)}{1+n(\mu)}, \mathrm{n}(\mu)$ and $\mathrm{n}(\mathrm{u})$ are the numbers of $\mu$ 's and u's satisfying $\left\{\mu: \mu_{l}\left(a_{i}, b_{j}\right) \geq \mu_{j}\right\}$ and $\left\{u: u_{l}\left(a_{i}, b_{j}\right) \geq u_{j}, \mu_{l}\left(a_{i}, b_{j}\right) \geq \mu_{j}\right\}$,

Step 4: Choice value $\left(c_{i}\right)=\sum_{j} d\left(a_{i}, b_{j}\right)$ for each i.

Step 5: Identify $c_{k}=\max \left\{c_{i}\right\}$. Obviously, optimal decision is to choose $a_{k}$. The optimal alternative, clearly followed by the definition of mid level soft set

Step 6: If $\mathrm{k}$ has multiple values, any one of $a_{k}$ may be selected.

Now in the next section, we demonstrate the pseudo code of the above algorithm.

\section{Pseudo code of the proposed algorithm}

Step 1: Start.

Step 2: Get the number of parameters and the number of objects, respectively. $[\mathrm{m}, \mathrm{n}]$

Step 3: Input the primary and secondary membership values for each parameter and object pairs. $\left[u_{l}\left(a_{i}, b_{j}\right)\right.$, $\left.\mu_{l}\left(a_{i}, b_{j}\right)\right]$

Step 4: Add the primary membership values to the running total. [ $\left.\sum u_{l}\left(a_{i}, b_{j}\right)\right]$

Step 5: Add the secondary membership value to a separate running total. [ $\left.\sum \mu_{l}\left(a_{i}, b_{j}\right)\right]$

Step 6: Increment the count of numbers read in for each parameter. [1]

Step 7: Calculate the average primary membership values by dividing the value obtained in step 4 by the value obtained in step 6. $\left[u_{j}=\frac{1}{l} \sum u_{l}\left(a_{i}, b_{j}\right)\right]$

Step 8: Calculate the average secondary membership values by dividing the value obtained in step 5 by the value obtained in step 6. $\left[\mu_{j}=\frac{1}{l} \sum \mu_{l}\left(a_{i}, b_{j}\right)\right]$

Step 9: Count the number of secondary membership values given in step 3, which are greater than or equal to the average secondary membership value obtained in step 8. $[n(\mu)]$

Step 10: Count the number of primary membership values given in step 3 , which are greater than or equal to 
the average primary membership value obtained in step 7 and also fulfill the condition in step 9. [n(u)]

Step 11: Add the value obtained in step 9 by $1 .[1+n(\mu)]$

Step 12: Divide the value obtained in step 10 by the value obtained in step 11. $\left[d\left(a_{i}, b_{j}\right)=\frac{n(u)}{1+n(\mu)}\right]$

Step 13: Do repeat step 9 to step 12 in $\mathrm{n}$ (number of objects) times.

Step 14: Do repeat step 3 to step 13 in m (number of parameters) times.

Step 15: Add the values obtained in step 12 to the new running total. $\left[c_{i}=\sum_{j} d\left(a_{i}, b_{j}\right)\right]$

Step 16: Do repeat step 15 in $\mathrm{n}$ (number of objects) times.

Step 17: Call the first number of the list obtained in step 15 as 'max'. $\left[\max =c_{1}\right]$

Step 18: Pick the next number in the list obtained in step 15.

Step 19: If it is bigger than ' $m a x$ ', replace the value of ' $m a x$ ' with the larger value and go to step 20. otherwise, go to step 18 .

Step 20: Identify the rank of 'max' obtained in step 19 in the list obtained in step 15. For multiple ranked, select any one of them. [k for $\left.\max =c_{k}\right]$

Step 21: Conclude that the k-th object is the optimal object. Step 22: Stop.

\section{Numerical example for T2FSS based DMP}

Now, we demonstrate the functioning of proposed algorithm with the help of numerical examples.

Example 15 Let $V=\left\{a_{1}, a_{2}, a_{3}, a_{4}, a_{5}, a_{6}\right\}$ be the set of six cars and $P=\{$ expensive, fuel efficiency, spacious, maintenance-free, eco friendly, security measure, automatic gear, manual gear\} be a set of parameters. Let Mr. X is interested in buying a car based on his choice parameters "expensive, fuel efficiency, spacious, eco friendly and security measure". Suppose the subset $Q=$ \{expensive cars, fuel efficiency cars, spacious cars, eco-friendly cars, security measure cars $\}=\left\{b_{1}, b_{2}, b_{3}, b_{4}, b_{5}\right\}$ of $\mathrm{P}$, where $b_{1}, b_{2}, b_{3}, b_{4}$, and $b_{5}$ stand for expensive cars, fuel efficiency cars, spacious cars, eco-friendly cars, and security measure cars respectively. Suppose the manufacturer car company provide some linguistic terms corresponding to each car, for each parameter chosen by Mr. X such as,

$$
\begin{aligned}
\mathcal{F}\left(b_{1}\right)= & \left\{\frac{\text { very expensive }}{a_{1}}, \frac{\text { very expensive }}{a_{2}},\right. \\
& \frac{\text { moderately inexpensive }}{a_{3}}, \frac{\text { very expensive }}{a_{4}}, \\
& \left.\frac{\text { inexpensive }}{a_{5}}, \frac{\text { moderately expensive }}{a_{6}}\right\}, \\
\mathcal{F}\left(b_{2}\right)= & \left\{\frac{\text { high fuel efficiency }}{a_{1}},\right.
\end{aligned}
$$

$$
\begin{aligned}
& \frac{\text { very high fuel efficiency }}{a_{2}}, \\
& \frac{\text { low fuel efficiency }}{a_{3}} \text {, } \\
& \frac{\text { low fuel efficiency }}{a_{4}} \text {, } \\
& \frac{\text { moderately low fuel efficiency }}{a_{5}}, \\
& \left.\frac{\text { moderately low fuel efficiency }}{a_{6}}\right\}, \\
& \mathcal{F}\left(b_{3}\right)=\left\{\frac{\text { very high spacious }}{a_{1}}, \frac{\text { moderately not spacious }}{a_{2}},\right. \\
& \frac{\text { very high spacious }}{a_{3}}, \frac{\text { very high specious }}{a_{4}}, \\
& \left.\frac{\text { moderately not spacious }}{a_{5}}, \frac{\text { not spacious }}{a_{6}}\right\} \\
& \mathcal{F}\left(b_{4}\right)=\left\{\frac{\text { low eco friendly }}{a_{1}}, \frac{\text { high eco friendly }}{a_{2}},\right. \\
& \frac{\text { moderately low eco friendly }}{a_{3}}, \\
& \frac{\text { high eco friendly }}{a_{4}}, \frac{\text { average eco friendly }}{a_{5}}, \\
& \left.\frac{\text { moderately low eco friendly }}{a_{6}}\right\} \\
& \mathcal{F}\left(b_{5}\right)=\left\{\frac{\text { average security measure }}{a_{1}},\right. \\
& \frac{\text { very high security measure }}{a_{2}}, \\
& \frac{\text { high security measure }}{a_{3}}, \\
& \frac{\text { average security measure }}{a_{4}}, \frac{\text { low security measure }}{a_{5}}, \\
& \left.\frac{\text { very high security measure }}{a_{6}}\right\}
\end{aligned}
$$

To choose the suitable car according to Mr.X's choice parameters, we first transfer the linguistic terms into the corresponding fuzzy sets, according to the Figs. 6, 7, 8, 9, 10, and then we apply the Algorithm 6.

After the conversion we have the T2FSS such as,

$$
\begin{aligned}
& (\mathcal{F}, Q)=\left\{b_{1}=\left\{\frac{\left\{\frac{0.6}{0.8}, \frac{0.8}{0.9}, \frac{0.9}{1.0}\right\}}{a_{1}}, \frac{\left\{\frac{0.6}{0.8}, \frac{0.8}{0.9}, \frac{0.9}{1.0}\right\}}{a_{2}},\right.\right. \\
& \frac{\left\{\frac{0.8}{0.1}, \frac{0.5}{0.2}\right\}}{a_{3}}, \frac{\left\{\frac{0.6}{0.8}, \frac{0.8}{0.9}, \frac{0.9}{1.0}\right\}}{a_{4}}, \frac{\left\{\frac{0.6}{0.1}\right\}}{a_{5}}, \\
& \left.\frac{\left\{\frac{0.5}{0.5}, \frac{0.6}{0.6}, \frac{0.9}{0.7}, \frac{1.0}{0.8}\right\}}{a_{6}}\right\} \text {, } \\
& b_{2}=\left\{\frac{\left\{\frac{0.4}{0.5}, \frac{0.6}{0.6}, \frac{0.8}{0.7}, \frac{0.5}{0.8}\right\}}{a_{1}}, \frac{\left\{\frac{0.5}{0.8}, \frac{0.9}{0.9}, \frac{0.7}{1.0}\right\}}{a_{2}},\right.
\end{aligned}
$$




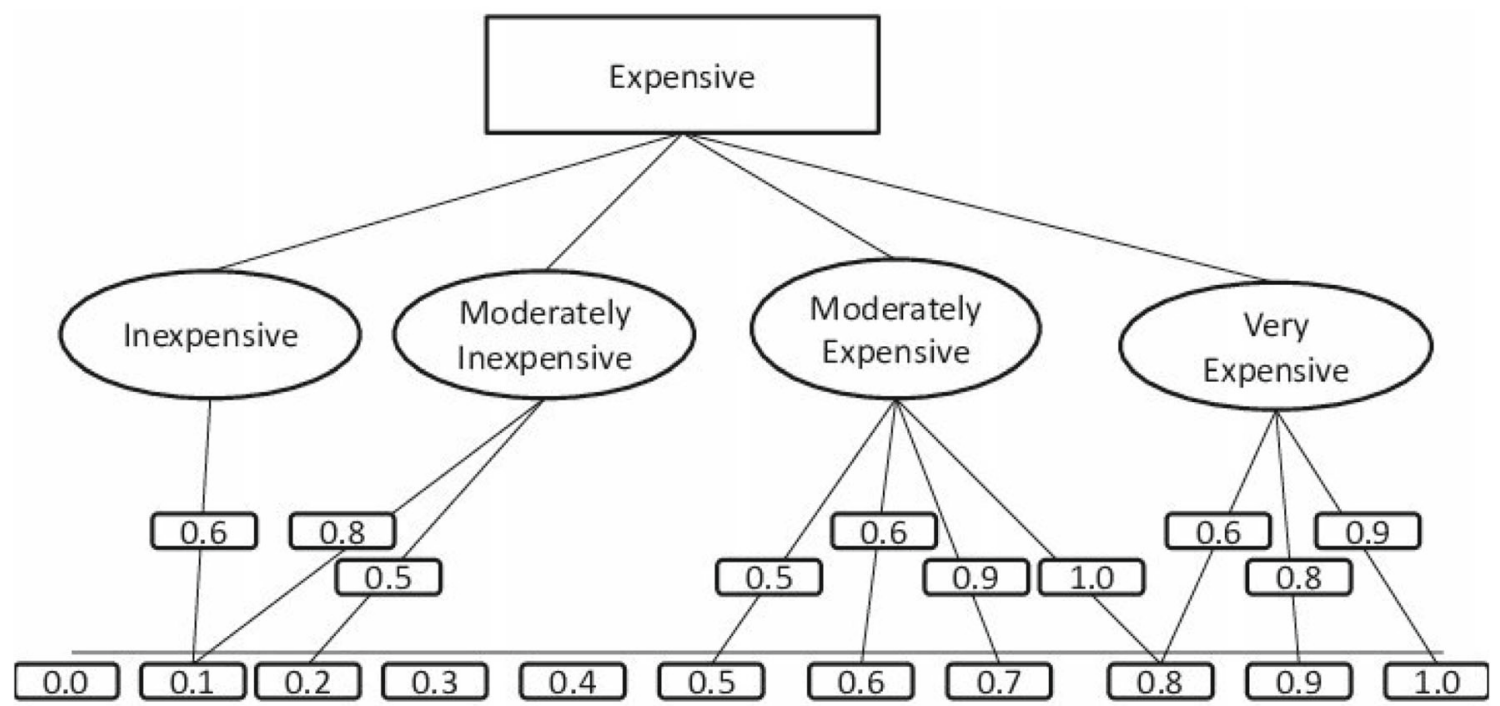

Fig. 6 Linguistic variable 'expensive'

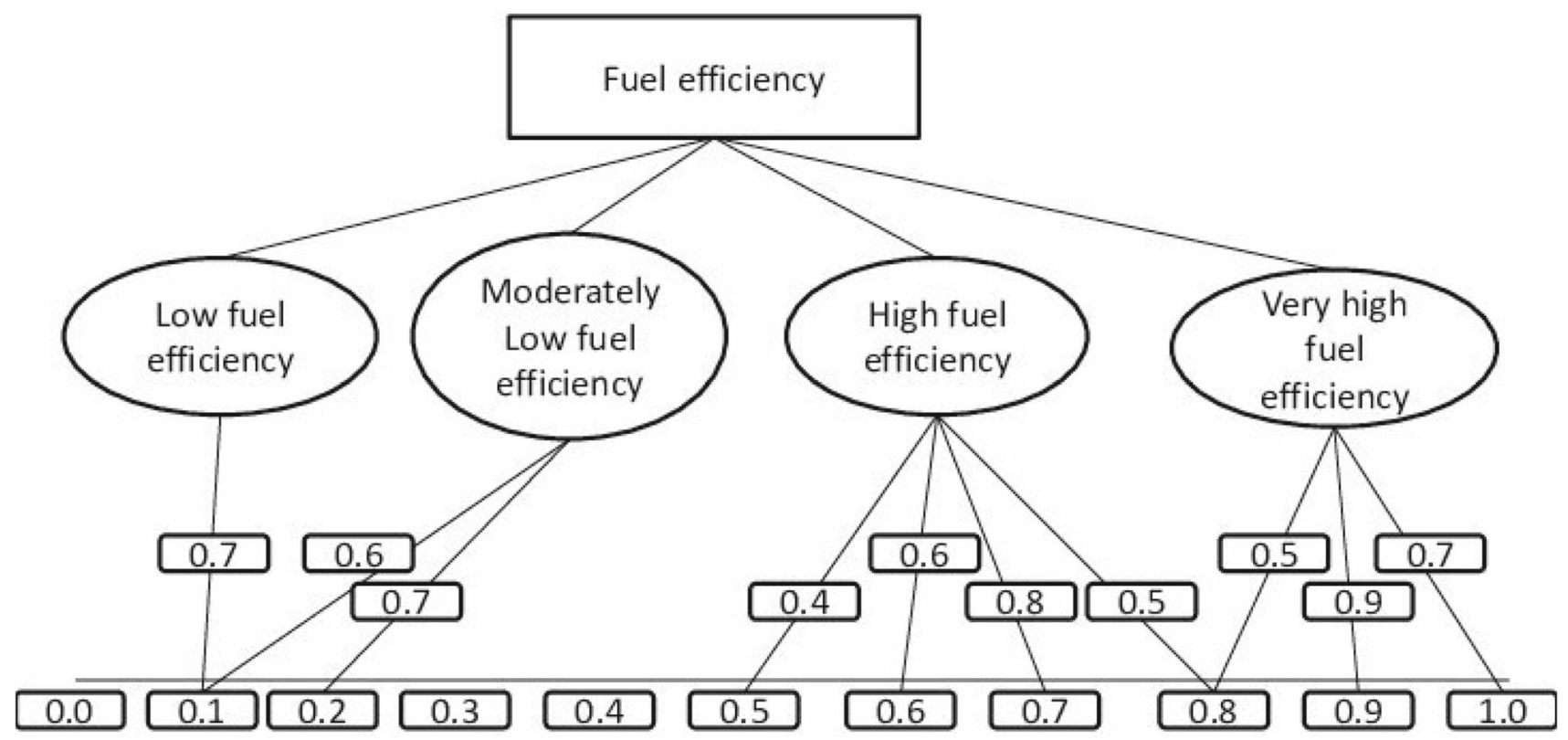

Fig. 7 Linguistic variable 'fuel efficiency'

$$
\begin{aligned}
& \left.\frac{\left\{\frac{0.7}{0.1}\right\}}{a_{3}}, \frac{\left\{\frac{0.7}{0.1}\right\}}{a_{4}}, \frac{\left\{\frac{0.6}{0.1}, \frac{0.7}{0.2}\right\}}{a_{5}}, \frac{\left\{\frac{0.6}{0.1}, \frac{0.7}{0.2}\right\}}{a_{6}}\right\}, \quad b_{4}=\left\{\frac{\left\{\frac{0.5}{0.1}\right\}}{a_{1}}, \frac{\left\{\frac{0.4}{0.8}, \frac{0.9}{0.9}, \frac{0.7}{1.0}\right\}}{a_{2}}, \frac{\left\{\frac{0.7}{0.1}, \frac{0.3}{0.2}\right\}}{a_{3}},\right. \\
& b_{3}=\left\{\frac{\left\{\frac{1.0}{0.8}, \frac{0.8}{0.9}, \frac{0.5}{1.0}\right\}}{a_{1}}, \frac{\left\{\frac{0.5}{0.1}, \frac{0.9}{0.2}\right\}}{a_{2}}, \frac{\left\{\frac{1.0}{0.8}, \frac{0.8}{0.9}, \frac{0.5}{1.0}\right\}}{a_{3}}, \quad \frac{\left\{\frac{0.4}{0.8}, \frac{0.9}{0.9}, \frac{0.7}{1.0}\right\}}{a_{4}}, \frac{\left\{\frac{0.5}{0.5}, \frac{0.6}{0.6}, \frac{0.7}{0.7}, \frac{0.4}{0.8}\right\}}{a_{5}},\right. \\
& \left.\left.\frac{\left\{\frac{1.0}{0.8}, \frac{0.8}{0.9}, \frac{0.5}{1.0}\right\}}{a_{4}}, \frac{\left\{\frac{0.5}{0.1}, \frac{0.9}{0.2}\right\}}{a_{5}}, \frac{\left\{\frac{0.8}{0.1}\right\}}{a_{6}}\right\}, \quad \frac{\left\{\frac{0.7}{0.1}, \frac{0.3}{0.2}\right\}}{a_{6}}\right\},
\end{aligned}
$$




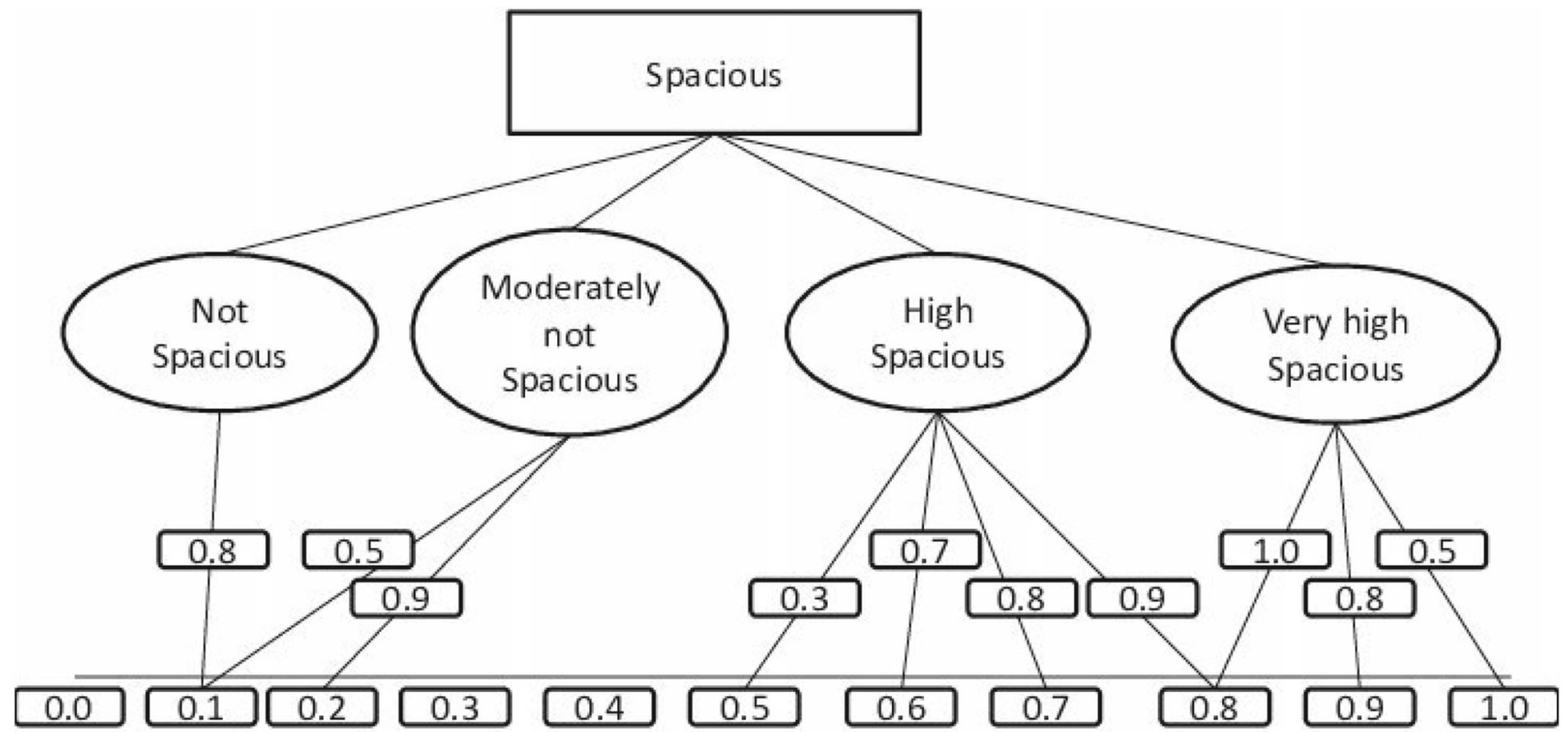

Fig. 8 Linguistic variable 'spacious'

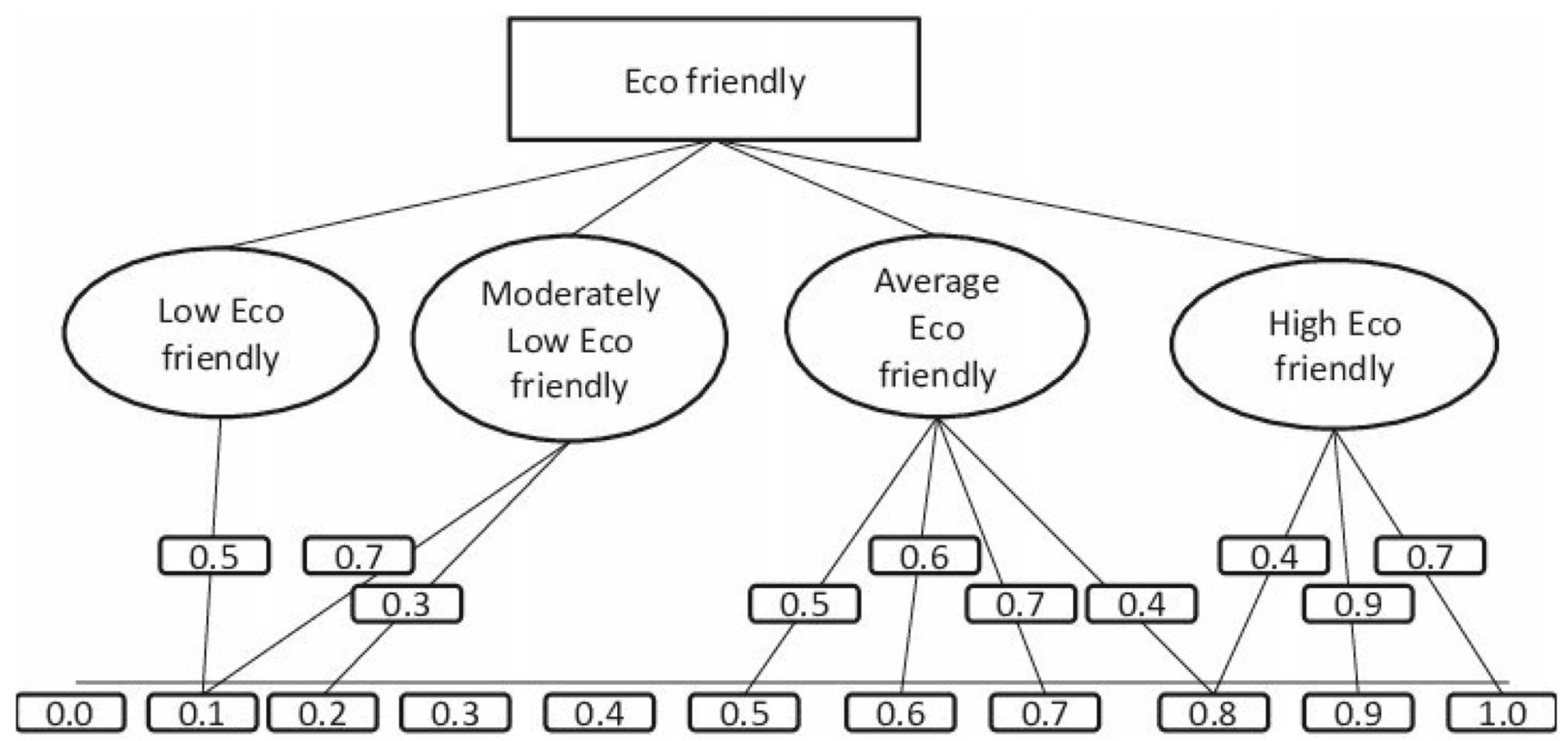

Fig. 9 Linguistic variable 'eco friendly'

$$
\begin{aligned}
& b_{5}=\left\{\frac{\left\{\frac{0.6}{0.2}, \frac{0.8}{0.3}\right\}}{a_{1}}, \frac{\left\{\frac{0.5}{0.8}, \frac{0.8}{0.9}, \frac{0.9}{1.0}\right\}}{a_{2}},\right. \\
& \frac{\left\{\frac{0.4}{0.5}, \frac{0.7}{0.6}, \frac{0.7}{0.7}, \frac{0.6}{0.8}\right\}}{a_{3}}, \\
& \left.\left.\frac{\left\{\frac{0.6}{0.2}, \frac{0.8}{0.3}\right\}}{a_{4}}, \frac{\left\{\frac{0.8}{0.1}\right\}}{a_{5}}, \frac{\left\{\frac{0.5}{0.8}, \frac{0.8}{0.9}, \frac{0.9}{1.0}\right\}}{a_{6}}\right\}\right\}
\end{aligned}
$$

Now we follow the steps in Algorithm 6 as,

Step 1: The tabular representation of the $\operatorname{T} 2 \mathrm{FSS}(\mathcal{F}, Q)$ is shown in Table 5.

Step 2: Two threshold fuzzy sets $\alpha$ and $\lambda: Q \rightarrow[0,1]$ are calculated as,

$$
\mu_{\alpha\left(b_{1}\right)}=0.74, \mu_{\alpha\left(b_{2}\right)}=0.65, \mu_{\alpha\left(b_{3}\right)}=0.75 \text {, }
$$




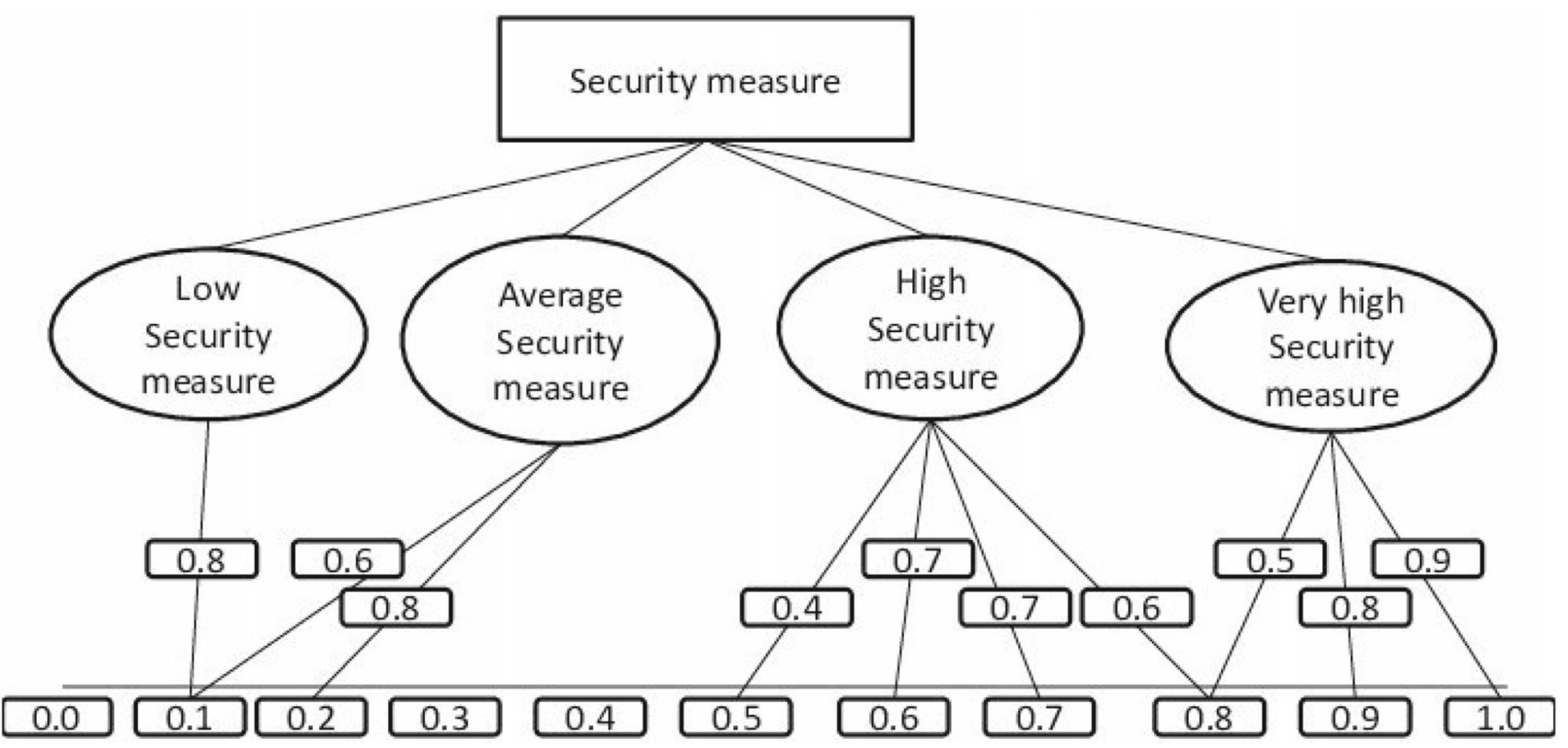

Fig. 10 Linguistic variable 'security measure'

Table 5 T2FSS $(\mathcal{F}, Q)$ in tabular representation

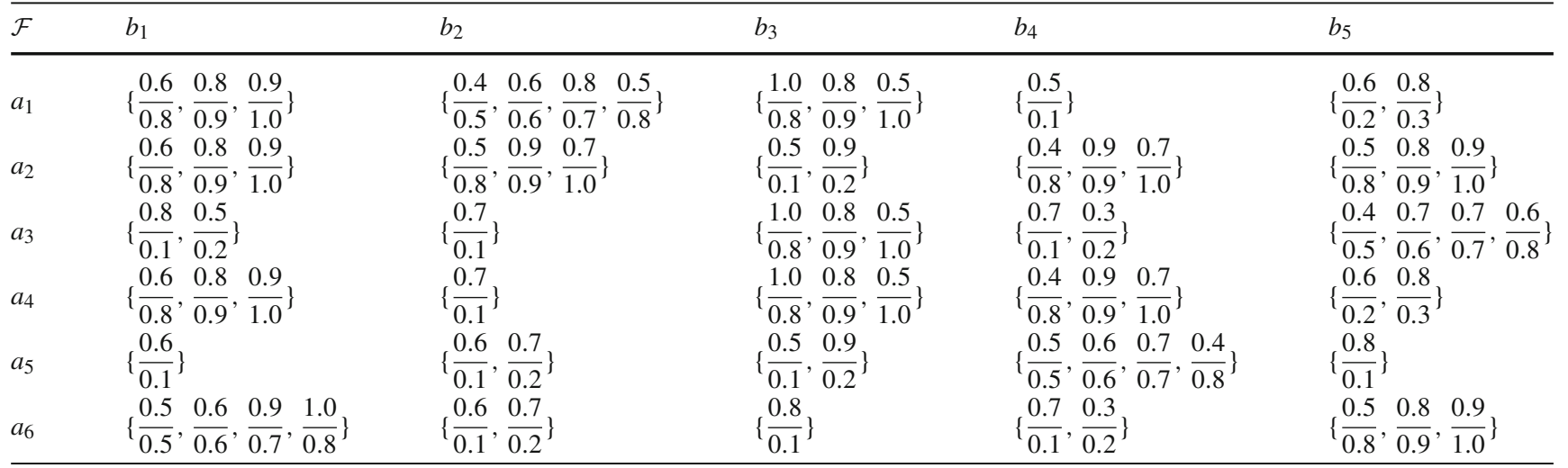

$$
\mu_{\alpha\left(b_{4}\right)}=0.58, \mu_{\alpha\left(b_{5}\right)}=0.69
$$

Therefore, $\alpha=\left\{\frac{0.74}{b_{1}}, \frac{0.65}{b_{2}}, \frac{0.75}{b_{3}}, \frac{0.58}{b_{4}}, \frac{0.69}{b_{5}}\right\}$.

$$
\begin{aligned}
& \mu_{\lambda\left(b_{1}\right)}=0.69, \mu_{\lambda\left(b_{2}\right)}=0.47, \mu_{\lambda\left(b_{3}\right)}=0.63, \\
& \mu_{\lambda\left(b_{4}\right)}=0.58, \mu_{\lambda\left(b_{5}\right)}=0.61 .
\end{aligned}
$$

Therefore, $\lambda=\left\{\frac{0.69}{b_{1}}, \frac{0.47}{b_{2}}, \frac{0.63}{b_{3}}, \frac{0.58}{b_{4}}, \frac{0.61}{b_{5}}\right\}$

Step 3: The LFSS $L((\mathcal{F}, Q) ; \alpha, \lambda)$ of $(\mathcal{F}, Q)$ with respect to the threshold fuzzy sets $\alpha$ and $\lambda$ is calculated as,

$$
\begin{aligned}
& \mathcal{F}\left(b_{1}\right)_{0.69}^{0.74}=\left\{\left(a_{1}, 0.67\right),\left(a_{2}, 0.67\right),\right.\left.\left(a_{4}, 0.67\right),\left(a_{6}, 0.67\right)\right\}, \\
& \mathcal{F}\left(b_{2}\right)_{0.47}^{0.65}=\left\{\left(a_{1}, 0.50\right),\left(a_{2}, 0.67\right)\right\},
\end{aligned}
$$

$$
\begin{aligned}
\mathcal{F}\left(b_{3}\right)_{0.63}^{0.75} & =\left\{\left(a_{1}, 0.67\right),\left(a_{3}, 0.67\right),\left(a_{4}, 0.67\right)\right\} \\
\mathcal{F}\left(b_{4}\right)_{0.58}^{0.58} & =\left\{\left(a_{2}, 0.67\right),\left(a_{4}, 0.67\right),\left(a_{5}, 0.67\right)\right\} \\
\mathcal{F}\left(b_{5}\right)_{0.61}^{0.69} & =\left\{\left(a_{2}, 0.67\right),\left(a_{3}, 0.33\right),\left(a_{6}, 0.67\right)\right\}
\end{aligned}
$$

Therefore, the $(\alpha, \lambda)$-level fuzzy soft set of $(\mathcal{F}, Q)$ is

$$
\begin{aligned}
L((\mathcal{F}, Q) ; \alpha, \lambda) & =\left\{b_{1}=\left\{\frac{0.67}{a_{1}}, \frac{0.67}{a_{2}}, \frac{0.67}{a_{4}}, \frac{0.67}{a_{6}}\right\},\right. \\
b_{2} & =\left\{\frac{0.50}{a_{1}}, \frac{0.67}{a_{2}}\right\} \\
b_{3} & =\left\{\frac{0.67}{a_{1}}, \frac{0.67}{a_{3}}, \frac{0.67}{a_{4}}\right\}, \\
b_{4} & =\left\{\frac{0.67}{a_{2}}, \frac{0.67}{a_{4}}, \frac{0.67}{a_{5}}\right\},
\end{aligned}
$$


Table $6(\alpha, \lambda)$-level fuzzy soft set $((\mathcal{F}, Q) ; \alpha, \lambda)$ in tabular form

\begin{tabular}{lllllll}
\hline $\mathcal{F}$ & $b_{1}$ & $b_{2}$ & $b_{3}$ & $b_{4}$ & $b_{5}$ & Choice values $u_{i}$ \\
\hline$a_{1}$ & 0.67 & 0.50 & 0.67 & 0 & 0 & $u_{1}=1.84$ \\
$a_{2}$ & 0.67 & 0.67 & 0 & 0.67 & 0.67 & $u_{2}=2.68$ \\
$a_{3}$ & 0 & 0 & 0.67 & 0 & 0.33 & $u_{3}=1$ \\
$a_{4}$ & 0.67 & 0 & 0.67 & 0.67 & 0 & $u_{4}=2.01$ \\
$a_{5}$ & 0 & 0 & 0 & 0.67 & 0 & $u_{5}=0.67$ \\
$a_{6}$ & 0.67 & 0 & 0 & 0 & 0.67 & $u_{6}=1.34$ \\
\hline
\end{tabular}

$$
\left.b_{5}=\left\{\frac{0.67}{a_{2}}, \frac{0.33}{a_{3}}, \frac{0.67}{a_{6}}\right\}\right\}
$$

Step 4: The mid-level fuzzy soft set $L((\mathcal{F}, Q) ; \alpha, \lambda)$ with choice values evaluated in Table 6 .

Step 5: Here $u_{2}=\max _{a_{i} \in V}\left\{u_{i}\right\}=2.68$ corresponding to $a_{2}$. Therefore we select $a_{2}$ as an optimal solution.

Example 16 Suitable location selection plays a very significant role in improving economic development and enhancing the stability of a company's existence. In several types of plants, such as fuel plants, industrial power plants, etc., it is very difficult to reverse the location deciding on plant construction location. Therefore, a company must select a suitable location for constructing a manufacturing plant where the workers would produce the goods. In this regard, based on available parameters such as watercourse easements, high voltage power line easements, distance to upper hierarchy roads, nature of the surface area, consistency of government policy, distance to train station etc., a group of three experts has been appointed in choosing the best location among four locations selected by the company.

Now assume that $U=\left\{a_{1}, a_{2}, a_{3}, a_{4}, a_{5}, a_{6}\right\}$ be a set of six places and $\mathrm{Q}=$ watercourse easements $\left(b_{1}\right)$, high voltage power line easements $\left(b_{2}\right)$, distance to upper hierarchy roads $\left(b_{3}\right)$, nature of the surface area $\left(b_{4}\right)$, consistency of government policy $\left(b_{5}\right)$ be a set of five associated parameters with respect to the elements of $\mathrm{U}$. Let an Industrial company $\mathrm{X}$ is interested to choose a suitable location based on their choice parameters listed above.

To choose an appropriate location according to the company $\mathrm{X}$ 's choice parameters, the decision maker first construct a T2FSS as,

$$
\begin{aligned}
(\mathcal{F}, Q)= & \left\{b_{1}=\left\{\frac{\left\{\frac{0.7}{0.7}, \frac{0.8}{0.9}, \frac{0.9}{1.0}\right\}}{a_{1}}, \frac{\left\{\frac{0.6}{0.6}, \frac{0.7}{0.9}, \frac{0.9}{1.0}\right\}}{a_{2}},\right.\right. \\
& \frac{\left\{\frac{0.9}{0.1}, \frac{0.5}{0.3}\right\}}{a_{3}},
\end{aligned}
$$

$$
\begin{aligned}
& \frac{\left\{\frac{0.7}{0.8}, \frac{0.7}{0.9}, \frac{0.9}{1.0}\right\}}{a_{4}}, \frac{\left\{\frac{0.9}{0.2}\right\}}{a_{5}}, \\
& \left.\frac{\left\{\frac{0.5}{0.5}, \frac{0.8}{0.6}, \frac{0.9}{0.7}, \frac{1.0}{0.9}\right\}}{a_{6}}\right\}, \\
& b_{2}=\left\{\frac{\left\{\frac{0.1}{0.4}, \frac{0.6}{0.6}, \frac{0.8}{0.7}, \frac{0.5}{0.8}\right\}}{a_{1}}, \frac{\left\{\frac{0.3}{0.8}, \frac{0.9}{0.9}, \frac{0.1}{1.0}\right\}}{a_{2}},\right. \\
& \frac{\left\{\frac{0.1}{0.2}\right\}}{a_{3}} \\
& \left.\frac{\left\{\frac{0.2}{0.3}\right\}}{a_{4}}, \frac{\left\{\frac{0.1}{0.1}, \frac{0.7}{0.3}\right\}}{a_{5}}, \frac{\left\{\frac{0.2}{0.1}, \frac{0.7}{0.4}\right\}}{a_{6}}\right\} \text {, } \\
& b_{3}=\left\{\frac{\left\{\frac{1.0}{0.7}, \frac{0.8}{0.9}, \frac{0.9}{1.0}\right\}}{a_{1}}, \frac{\left\{\frac{0.8}{0.2}, \frac{0.9}{0.3}\right\}}{a_{2}}, \frac{\left\{\frac{1.0}{0.6}, \frac{0.8}{0.9}, \frac{0.9}{1.0}\right\}}{a_{3}},\right. \\
& \left.\frac{\left\{\frac{1.0}{0.5}, \frac{0.8}{0.9}, \frac{0.7}{1.0}\right\}}{a_{4}}, \frac{\left\{\frac{0.8}{0.1}, \frac{0.9}{0.3}\right\}}{a_{5}}, \frac{\left\{\frac{0.9}{0.2}\right\}}{a_{6}}\right\}, \\
& b_{4}=\left\{\frac{\left\{\frac{0.1}{0.2}\right\}}{a_{1}}, \frac{\left\{\frac{0.4}{0.6}, \frac{0.9}{0.8}, \frac{0.2}{1.0}\right\}}{a_{2}}, \frac{\left\{\frac{0.7}{0.1}, \frac{0.1}{0.3}\right\}}{a_{3}},\right. \\
& \frac{\left\{\frac{0.3}{0.6}, \frac{0.9}{0.7}, \frac{0.7}{0.9}\right\}}{a_{4}}, \frac{\left\{\frac{0.1}{0.1}, \frac{0.6}{0.6}, \frac{0.7}{0.7}, \frac{0.4}{0.8}\right\}}{a_{5}} \\
& \left.\frac{\left\{\frac{0.7}{0.1}, \frac{0.1}{0.3}\right\}}{a_{6}}\right\} \text {, } \\
& b_{5}=\left\{\frac{\left\{\frac{0.2}{0.1}, \frac{0.9}{0.4}\right\}}{a_{1}}, \frac{\left\{\frac{0.1}{0.6}, \frac{0.8}{0.8}, \frac{0.9}{1.0}\right\}}{a_{2}}\right. \text {, } \\
& \frac{\left\{\frac{0.1}{0.4}, \frac{0.9}{0.6}, \frac{0.7}{0.7}, \frac{0.6}{0.9}\right\}}{a_{3}}, \\
& \left.\left.\frac{\left\{\frac{0.2}{0.1}, \frac{0.8}{0.4}\right\}}{a_{4}}, \frac{\left\{\frac{0.7}{0.4}\right\}}{a_{5}}, \frac{\left\{\frac{0.1}{0.6}, \frac{0.8}{0.8}, \frac{0.7}{1.0}\right\}}{a_{6}}\right\}\right\}
\end{aligned}
$$

Now we follow the steps in Algorithm 6 as,

Step 1: The tabular representation of the $\operatorname{T} 2 \mathrm{FSS}(\mathcal{F}, Q)$ is shown in Table 7.

Step 2: Two threshold fuzzy sets $\alpha$ and $\lambda: Q \rightarrow[0,1]$ are calculated as,

$\mu_{\alpha\left(b_{1}\right)}=0.78, \mu_{\alpha\left(b_{2}\right)}=0.41, \mu_{\alpha\left(b_{3}\right)}=0.87$, $\mu_{\alpha\left(b_{4}\right)}=0.46, \mu_{\alpha\left(b_{5}\right)}=0.57$ 
Table 7 T2FSS $(\mathcal{F}, Q)$ in tabular representation

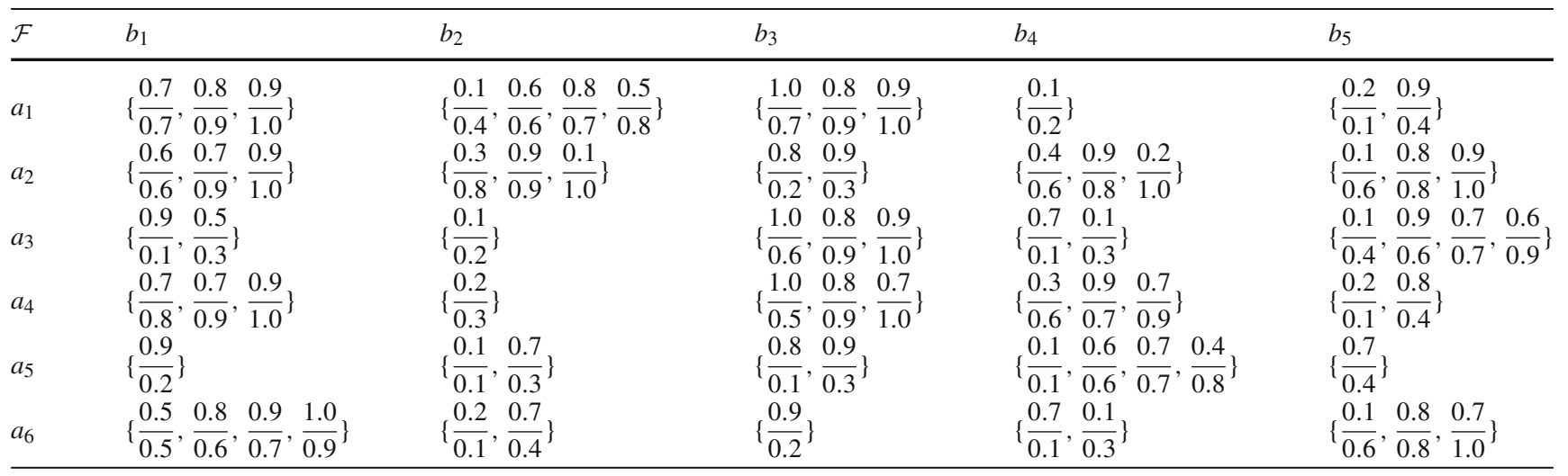

Therefore, $\alpha=\left\{\frac{0.78}{b_{1}}, \frac{0.41}{b_{2}}, \frac{0.87}{b_{3}}, \frac{0.46}{b_{4}}, \frac{0.57}{b_{5}}\right\}$.

$\mu_{\lambda\left(b_{1}\right)}=0.69, \mu_{\lambda\left(b_{2}\right)}=0.51, \mu_{\lambda\left(b_{3}\right)}=0.61$,

$\mu_{\lambda\left(b_{4}\right)}=0.52, \mu_{\lambda\left(b_{5}\right)}=0.59$.

Therefore, $\lambda=\left\{\frac{0.69}{b_{1}}, \frac{0.51}{b_{2}}, \frac{0.61}{b_{3}}, \frac{0.52}{b_{4}}, \frac{0.59}{b_{5}}\right\}$

Step 3: The LFSS $L((\mathcal{F}, Q) ; \alpha, \lambda)$ of $(\mathcal{F}, Q)$ with respect to the threshold fuzzy sets $\alpha$ and $\lambda$ is calculated as,

$$
\begin{aligned}
& \mathcal{F}\left(b_{1}\right)_{0.69}^{0.78}=\left\{\left(a_{1}, 0.67\right),\left(a_{2}, 0.50\right),\left(a_{4}, 0.50\right),\left(a_{6}, 0.50\right)\right\}, \\
& \mathcal{F}\left(b_{2}\right)_{0.51}^{0.41}=\left\{\left(a_{1}, 0.75\right),\left(a_{2}, 0.50\right)\right\} \\
& \mathcal{F}\left(b_{3}\right)_{0.61}^{0.87}=\left\{\left(a_{1}, 0.67\right),\left(a_{3}, 0.33\right)\right\} \\
& \mathcal{F}\left(b_{4}\right)_{0.52}^{0.46}=\left\{\left(a_{2}, 0.50\right),\left(a_{4}, 0.67\right),\left(a_{5}, 0.67\right)\right\} \\
& \mathcal{F}\left(b_{5}\right)_{0.59}^{0.57}=\left\{\left(a_{2}, 0.67\right),\left(a_{3}, 0.75\right),\left(a_{6}, 0.67\right)\right\}
\end{aligned}
$$

Therefore, the $(\alpha, \lambda)$-level fuzzy soft set of $(\mathcal{F}, Q)$ is

$$
\begin{aligned}
L((\mathcal{F}, Q) ; \alpha, \lambda) & =\left\{b_{1}=\left\{\frac{0.67}{a_{1}}, \frac{0.50}{a_{2}}, \frac{0.50}{a_{4}}, \frac{0.50}{a_{6}}\right\},\right. \\
b_{2} & =\left\{\frac{0.75}{a_{1}}, \frac{0.50}{a_{2}}\right\}, \\
b_{3} & =\left\{\frac{0.67}{a_{1}}, \frac{0.33}{a_{3}}\right\}, \\
b_{4} & =\left\{\frac{0.50}{a_{2}}, \frac{0.67}{a_{4}}, \frac{0.67}{a_{5}}\right\}, \\
b_{5} & \left.=\left\{\frac{0.67}{a_{2}}, \frac{0.75}{a_{3}}, \frac{0.67}{a_{6}}\right\}\right\}
\end{aligned}
$$

Step 4: The mid-level fuzzy soft set $L((\mathcal{F}, Q) ; \alpha, \lambda)$ with choice values evaluated in Table 8 .

Step 5: Here $u_{2}=\max _{a_{i} \in U}\left\{u_{i}\right\}=2.17$ corresponding to $a_{2}$. Therefore $a_{2}$ is the optimal choice location.
Table $8(\alpha, \lambda)$-level fuzzy soft set $((\mathcal{F}, Q) ; \alpha, \lambda)$ in tabular form

\begin{tabular}{lllllll}
\hline $\mathcal{F}$ & $b_{1}$ & $b_{2}$ & $b_{3}$ & $b_{4}$ & $b_{5}$ & Choice values $u_{i}$ \\
\hline$a_{1}$ & 0.67 & 0.75 & 0.67 & 0 & 0 & $u_{1}=2.09$ \\
$a_{2}$ & 0.50 & 0.50 & 0 & 0.50 & 0.67 & $u_{2}=2.17$ \\
$a_{3}$ & 0 & 0 & 0.33 & 0 & 0.75 & $u_{3}=1.08$ \\
$a_{4}$ & 0.50 & 0 & 0 & 0.67 & 0 & $u_{4}=1.17$ \\
$a_{5}$ & 0 & 0 & 0 & 0.67 & 0 & $u_{5}=0.67$ \\
$a_{6}$ & 0.50 & 0 & 0 & 0 & 0.67 & $u_{6}=1.17$ \\
\hline
\end{tabular}

\section{Proposed algorithm for WT2FSS based DMP}

Step 1: Provides T2FSS $(\mathcal{F}, Q)$.

Step 2: Find $\alpha$ and $\lambda$ according to the Definition 6 and Definition 7 , respectively.

Step 3: Calculate the LFSS $L((\mathcal{F}, Q) ; \alpha, \lambda)$ of $(\mathcal{F}, Q)$ w.r. to the threshold fuzzy sets $\alpha$ and $\lambda$.

Step 4: Prepare the tabular representation of the LFSS $L((\mathcal{F}, Q) ; \alpha, \lambda)$. For any $a_{i} \in V$, compute the weighted choice-value $u_{i}^{*}$ of $a_{i}$ where $u_{i}^{*}=\sum_{b \in Q}$ $\mathcal{F}(b)\left(a_{i}\right) . w(b)$.

Step 5: If $u_{k}^{*}=\max _{a_{i} \in V}\left\{u_{i}^{*}\right\}$ then $a_{k}$ is the optimal choice object.

Step 6: If $\mathrm{k}$ has more than one value, then any one of $a_{k}$ may be chosen.

\section{Numerical example for WT2FSS based DMP}

Example 17 Let us consider $\mathrm{V}=\left\{a_{1}, a_{2}, a_{3}, a_{4}, a_{5}, a_{6}\right\}$ be the collection of six houses, $\mathrm{P}=\left\{\right.$ expensive $=b_{1}$, beautiful $=b_{2}$, wooden $=b_{3}$, modern $=b_{4}$, in the green surrounding $=b_{5}$, cheap $=b_{6}$, in good repair $=b_{7}$, in average repair $\left.=b_{8}\right\}$ be the collection of parameters. Let Mr. X is interested to buy a car according to his choice parameters "expensive, beautiful, wooden, modern, in the green surrounding" which constitute 
the subset $\mathrm{Q}=\{$ expensive houses, beautiful houses, wooden houses, modern houses, in the green surrounding houses $\}=$ $\left\{b_{1}, b_{2}, b_{3}, b_{4}, b_{5}\right\}$ of the set P.

Consider the T2FSS $(\mathcal{F}, Q)$ which describes the 'attractiveness of the house' given by

$$
\begin{aligned}
& (\mathcal{F}, Q)=\left\{b_{1}=\left\{\frac{\left\{\frac{0.4}{0.2}, \frac{1.0}{0.3}, \frac{0.6}{0.4}\right\}}{a_{1}}, \frac{\left\{\frac{0.3}{0.4}, \frac{1.0}{0.5}, \frac{0.4}{0.7}\right\}}{a_{2}},\right.\right. \\
& \frac{\left\{\frac{0.7}{0.6}, \frac{1.0}{0.9}\right\}}{a_{3}}, \\
& \frac{\left\{\frac{0.4}{0.5}, \frac{0.8}{0.6}, \frac{0.3}{0.7}\right\}}{a_{4}}, \frac{\left\{\frac{0.6}{0.7}\right\}}{a_{5}}, \\
& \left.\frac{\left\{\frac{0.3}{0.2}, \frac{0.6}{0.6}, \frac{1.0}{0.7}, \frac{0.4}{0.8}\right\}}{a_{6}}\right\}, \\
& b_{2}=\left\{\frac{\left\{\frac{0.6}{0.2}, \frac{0.6}{0.5}, \frac{0.7}{0.6}, \frac{0.4}{0.7}\right\}}{a_{1}}, \frac{\left\{\frac{0.2}{0.2}, \frac{0.7}{0.3}, \frac{0.8}{0.5}\right\}}{a_{2}},\right. \\
& \frac{\left\{\frac{0.8}{0.9}\right\}}{a_{3}} \\
& \left.\frac{\left\{\frac{1.0}{0.5}\right\}}{a_{4}}, \frac{\left\{\frac{0.7}{0.6}, \frac{1.0}{0.7}\right\}}{a_{5}}, \frac{\left\{\frac{0.7}{0.2}, \frac{0.8}{0.5}\right\}}{a_{6}}\right\}, \\
& b_{3}=\left\{\frac{\left\{\frac{0.3}{0.7}, \frac{0.9}{0.8}, \frac{0.7}{0.9}\right\}}{a_{1}}, \frac{\left\{\frac{0.7}{0.5}, \frac{0.9}{0.8}\right\}}{a_{2}},\right. \\
& \underline{\left\{\frac{0.6}{0.4}, \frac{1.0}{0.5}, \frac{0.5}{0.7}\right\}}, \\
& a_{3} \\
& \left.\frac{\left\{\frac{0.4}{0.6}, \frac{1.0}{0.7}, \frac{0.5}{0.9}\right\}}{a_{4}}, \frac{\left\{\frac{0.7}{0.6}, \frac{1.0}{0.7}\right\}}{a_{5}}, \frac{\left\{\frac{1.0}{0.9}\right\}}{a_{6}}\right\}, \\
& b_{4}=\left\{\frac{\left\{\frac{0.6}{0.8}\right\}}{a_{1}}, \frac{\left\{\frac{0.4}{0.2}, \frac{0.9}{0.6}, \frac{0.6}{0.9}\right\}}{a_{2}}, \frac{\left\{\frac{0.8}{0.5}, \frac{0.9}{0.9}\right\}}{a_{3}},\right. \\
& \frac{\left\{\frac{0.3}{0.5}, \frac{0.8}{0.6}, \frac{0.6}{0.7}\right\}}{a_{4}}, \frac{\left\{\frac{0.8}{0.2}, \frac{0.7}{0.3}, \frac{0.4}{0.6}, \frac{0.4}{0.7}\right\}}{a_{5}}, \\
& \left.\frac{\left\{\frac{0.5}{0.6}, \frac{0.4}{0.8}\right\}}{a_{6}}\right\} \text {, } \\
& b_{5}=\left\{\frac{\left\{\frac{0.9}{0.6}, \frac{1.0}{0.9}\right\}}{a_{1}}, \frac{\left\{\frac{0.2}{0.2}, \frac{1.0}{0.6}, \frac{0.7}{0.7}\right\}}{a_{2}},\right.
\end{aligned}
$$

$$
\begin{aligned}
& \frac{\left\{\frac{0.4}{0.3}, \frac{0.8}{0.5}, \frac{0.9}{0.6}, \frac{1.0}{0.8}\right\}}{a_{3}}, \\
& \left.\left.\frac{\left\{\frac{0.8}{0.6}, \frac{0.9}{0.9}\right\}}{a_{4}}, \frac{\left\{\frac{0.8}{0.6}\right\}}{a_{5}}, \frac{\left\{\frac{0.3}{0.7}, \frac{0.6}{0.8}, \frac{0.5}{0.9}\right\}}{a_{6}}\right\}\right\} \text {. }
\end{aligned}
$$

Let Mr. X imposes the weights for the parameters in Q as: for the parameter "expensive", $w_{1}=0.31$;

for "beautiful", $w_{2}=0.24$;

for "wooden", $w_{3}=0.14$;

for "modern", $w_{4}=0.21$;

for "in the green surrounding", $w_{5}=0.10$.

Then we have a weight function $\mathrm{w}$ from $\mathrm{Q}$ to $[0,1]$ and the T2FSS $(\mathcal{F}, Q)$ is changed into a WT2FSS $(\mathcal{F}, Q, w)$.

Table 9 gives the tabular representation of $(\mathcal{F}, Q, w)$. The problem is to select the house which is most suitable for $\mathrm{Mr}$. $\mathrm{X}$.

Now we apply Algorithm 10 as,

Step 1: The tabular representation of the $\mathrm{WT} 2 \mathrm{FSS}(\mathcal{F}, Q, w)$ is given in Table 9.

Step 2: Two threshold fuzzy sets $\alpha$ and $\lambda: Q \rightarrow[0,1]$ are calculated as follows :

By the Definition 6,

$$
\begin{aligned}
& \mu_{\alpha\left(b_{1}\right)}=\frac{1}{16}[(0.4+1.0+0.6)+(0.3+1.0+0.4) \\
& +(0.7+1.0)+(0.4+0.8+0.3)+0.6 \\
& +(0.3+0.6+1.0+0.4)]=0.61
\end{aligned}
$$

Similarly, $\mu_{\alpha\left(b_{2}\right)}=0.70, \mu_{\alpha\left(b_{3}\right)}=0.73, \mu_{\alpha\left(b_{4}\right)}=0.61$, $\mu_{\alpha\left(b_{5}\right)}=0.72$

Therefore, $\alpha=\left\{\frac{0.61}{b_{1}}, \frac{0.70}{b_{2}}, \frac{0.73}{b_{3}}, \frac{0.61}{b_{4}}, \frac{0.72}{b_{5}}\right\}$

By the Definition 7, we get, $\mu_{\lambda\left(b_{1}\right)}=0.55, \mu_{\lambda\left(b_{2}\right)}=0.49$, $\mu_{\lambda\left(b_{3}\right)}=0.69, \mu_{\lambda\left(b_{4}\right)}=0.59, \mu_{\lambda\left(b_{5}\right)}=0.64$.

Therefore, $\lambda=\left\{\frac{0.55}{b_{1}}, \frac{0.49}{b_{2}}, \frac{0.69}{b_{3}}, \frac{0.59}{b_{4}}, \frac{0.64}{b_{5}}\right\}$

Step 3: The LFSS $L((\mathcal{F}, Q) ; \alpha, \lambda)$ of $(\mathcal{F}, Q)$ w.r. to the threshold fuzzy-sets $\alpha$ and $\lambda$ is

$$
\begin{aligned}
& L((\mathcal{F}, Q) ; \alpha, \lambda) \\
& \quad=\left\{b_{1}=\left\{\left(a_{3}, 0.67\right),\left(a_{4}, 0.5\right),\left(a_{6}, 0.5\right)\right\},\right. \\
& \quad b_{2}=\left\{\left(a_{1}, 0.5\right),\left(a_{2}, 0.33\right),\left(a_{3}, 0.5\right),\left(a_{4}, 0.5\right),\right. \\
& \left.\left(a_{5}, 0.67\right),\left(a_{6}, 0.33\right)\right\}, \\
& b_{3}=\left\{\left(a_{1}, 0.5\right),\left(a_{2}, 0.5\right),\left(a_{4}, 0.5\right),\left(a_{5}, 0.5\right),\left(h_{6}, 0.5\right)\right\}, \\
& b_{4}=\left\{\left(h_{2}, 0.5\right),\left(a_{3}, 0.33\right),\left(a_{4}, 0.5\right)\right\}, \\
& \left.b_{5}=\left\{\left(a_{1}, 0.33\right),\left(a_{3}, 0.25\right),\left(a_{4}, 0.33\right)\right\}\right\}
\end{aligned}
$$


Table 9 WT2FSS $(\mathcal{F}, Q)$ in tabular form

\begin{tabular}{|c|c|c|c|c|c|}
\hline $\mathcal{F}$ & $b_{1}, w_{1}=0.31$ & $b_{2}, w_{2}=0.24$ & $b_{3}, w_{3}=0.14$ & $b_{4}, w_{4}=0.21$ & $b_{5}, w_{5}=0.10$ \\
\hline & $\begin{array}{lll}0.4 & 1.0 & 0.6\end{array}$ & $\begin{array}{llll}0.6 & 0.6 & 0.7 & 0.4\end{array}$ & $\begin{array}{lll}0.3 & 0.9 & 0.7\end{array}$ & 0.6 & $0.9 \quad 1.0$ \\
\hline$a_{1}$ & $\{\overline{0.2}, \overline{0.3}, \overline{0.4}\}$ & $\{\overline{0.2}, \overline{0.5}, \overline{0.6}, \overline{0.7}\}$ & $\{\overline{0.7}, \overline{0.8}, \overline{0.9}\}$ & $\{\overline{0.8}\}$ & $\{\overline{0.6}, \overline{0.9}\}$ \\
\hline$a_{2}$ & $\left\{\begin{array}{lll}0.3 & 1.0 & 0.4\end{array}\right\}$ & $\left\{\begin{array}{lll}0.2 & 0.7 & 0.8\end{array}\right\}$ & $\left\{\begin{array}{ll}0.7 & 0.9\end{array}\right\}$ & $\left\{\begin{array}{lll}0.4 & 0.9 & 0.6\end{array}\right\}$ & $\left\{\begin{array}{lll}0.2 & 1.0 & 0.7\end{array}\right\}$ \\
\hline$u_{2}$ & $\overline{0.4}, \overline{0.5}, \overline{0.7}$ & $\overline{0.2}, \overline{0.3}, \overline{0.5}$ & $\{\overline{0.5}, \overline{0.8}\}$ & $\{\overline{0.2}, \overline{0.6}, \overline{0.9}\}$ & $\{\overline{0.2}, \overline{0.6}, \overline{0.7}\}$ \\
\hline$a_{3}$ & $\left\{\frac{0.7}{0.6}, \frac{1.0}{0.9}\right\}$ & $\left\{\frac{0.8}{0.9}\right\}$ & $\left\{\frac{0.6}{0.4}, \frac{1.0}{0.5}, \frac{0.5}{0.7}\right\}$ & $\left\{\frac{0.8}{0.5}, \frac{0.9}{0.9}\right\}$ & $\left\{\frac{0.4}{0.3}, \frac{0.8}{0.5}, \frac{0.9}{0.6}, \frac{1.0}{0.8}\right\}$ \\
\hline$a_{4}$ & $\left\{\frac{0.4}{0.5}, \frac{0.8}{0.6}, \frac{0.3}{0.7}\right\}$ & $\left\{\frac{1.0}{0.5}\right\}$ & $\left\{\frac{0.4}{0.6}, \frac{1.0}{0.7}, \frac{0.5}{0.9}\right\}$ & $\left\{\frac{0.3}{0.5}, \frac{0.8}{0.6}, \frac{0.6}{0.7}\right\}$ & $\left\{\frac{0.8}{0.6}, \frac{0.9}{0.9}\right\}$ \\
\hline$a_{5}$ & $\left\{\frac{0.6}{0.7}\right\}$ & $\left\{\frac{0.7}{0.6}, \frac{1.0}{0.7}\right\}$ & $\left\{\frac{0.7}{0.6}, \frac{1.0}{0.7}\right\}$ & $\left\{\frac{0.8}{0.2}, \frac{0.7}{0.3}, \frac{0.4}{0.6}, \frac{0.4}{0.7}\right\}$ & $\left\{\frac{0.8}{0.6}\right\}$ \\
\hline$a_{6}$ & $\left\{\frac{0.3}{0.2}, \frac{0.6}{0.6}, \frac{1.0}{0.7}, \frac{0.4}{0.8}\right\}$ & $\left\{\frac{0.7}{0.2}, \frac{0.8}{0.5}\right\}$ & $\left\{\frac{1.0}{0.9}\right\}$ & $\left\{\frac{0.5}{0.6}, \frac{0.4}{0.8}\right\}$ & $\left\{\frac{0.3}{0.7}, \frac{0.6}{0.8}, \frac{0.5}{0.9}\right\}$ \\
\hline
\end{tabular}

Table 10 Level soft set $((\mathcal{F}, Q) ; \alpha, \lambda)$ in tabular form

\begin{tabular}{lllllll}
\hline $\mathcal{F}$ & $b_{1}, w_{1}=0.31$ & $b_{2}, w_{2}=0.24$ & $b_{3}, w_{3}=0.14$ & $b_{4}, w_{4}=0.21$ & $b_{5}, w_{5}=0.10$ & Choice values $v_{i}^{*}$ \\
\hline$a_{1}$ & 0 & 0.5 & 0.5 & 0 & 0.33 & $u_{1}^{*}=0.223$ \\
$a_{2}$ & 0 & 0.33 & 0.5 & 0.5 & 0 & $u_{2}^{*}=0.254$ \\
$a_{3}$ & 0.67 & 0.5 & 0 & 0.33 & 0.25 & $u_{3}^{*}=0.422$ \\
$a_{4}$ & 0.5 & 0.5 & 0.5 & 0.5 & 0 & $u_{4}^{*}=0.483$ \\
$a_{5}$ & 0 & 0.67 & 0.5 & 0 & 0 & $u_{5}^{*}=0.231$ \\
$a_{6}$ & 0.5 & 0.33 & 0.5 & 0.33 & $u_{6}^{*}=0.304$ \\
\hline
\end{tabular}

Table 11 Level soft set $((\mathcal{F}, Q) ; \alpha, \lambda)$ in tabular form

\begin{tabular}{lllllll}
\hline $\mathcal{F}$ & $b_{1}, w_{1}=0.31$ & $b_{2}, w_{2}=0.24$ & $b_{3}, w_{3}=0.14$ & $b_{4}, w_{4}=0.21$ & $b_{5}, w_{5}=0.10$ & Choice values $\bar{c}_{i}$ \\
\hline$a_{1}$ & 0 & 0 & 1 & 0 & 0 & $\bar{c}_{1}=0.14$ \\
$a_{2}$ & 0 & 0 & 1 & 1 & 0 & $\bar{c}_{2}=0.35$ \\
$a_{3}$ & 1 & 1 & 0 & 1 & 0 & $\bar{c}_{3}=0.76$ \\
$a_{4}$ & 1 & 0 & 1 & 1 & 0 & $\bar{c}_{4}=0.76$ \\
$a_{5}$ & 1 & 1 & 1 & 0 & 0 & $\bar{c}_{5}=0.69$ \\
$a_{6}$ & 1 & 0 & 1 & 0 & $\bar{c}_{6}=0.45$ \\
\hline
\end{tabular}

Step 4: The mid-level fuzzy-soft-set $L((\mathcal{F}, Q) ; \alpha, \lambda)$ with weighted choice values given in Table 10.

Step 5: $u_{4}^{*}=\max _{a_{i} \in V}\left\{u_{i}^{*}\right\}=0.483$ corresponding to $a_{4}$. Therefore $a_{4}$ is the optimal choice object.

\section{Comparison}

Let us consider the above numerical example and try to solve it by Z. Zhang's method.

Step 1: Input T2FSS $(\mathcal{F}, Q)$ with weights of each parameters (Given in Sect. 11).
Step 2: Input two threshold fuzzy sets

$$
\begin{aligned}
& \alpha=\left\{\frac{0.6}{b_{1}}, \frac{0.6}{b_{2}}, \frac{0.8}{b_{3}}, \frac{0.7}{b_{4}}, \frac{0.9}{b_{5}}\right\} \text { and } \\
& \lambda=\left\{\frac{0.5}{b_{1}}, \frac{0.6}{b_{2}}, \frac{0.7}{b_{3}}, \frac{0.5}{b_{4}}, \frac{0.7}{b_{5}}\right\}
\end{aligned}
$$

Step 3: Compute the level soft set $L((\mathcal{F}, Q) ; \alpha, \lambda))$ according to Definition 4, as shown in Table 11.

Step 4: Compute the weighted choice-value $\bar{c}_{i}$ of $a_{i}$. The tabular representation of $L((\mathcal{F}, Q) ; \alpha, \lambda))$ with weighted choice values is shown in Table 11.

Step 5: Since $\operatorname{Max} 1 \leq i \leq 6\left\{\bar{c}_{i}\right\}=\left\{\bar{c}_{3}, \bar{c}_{4}\right\}$, so $a_{3}$ or $a_{4}$ is the optimal choice object.

Zhang's method fails to determine a unique solution and partially agrees with the result of our proposed method shown above (Sect. 11). On the other hand, if the decision-maker 
chooses the different threshold fuzzy sets $\alpha^{\prime}$ and $\lambda^{\prime}$, then the optimal objective will be changed. So we claimed that our proposed approach is more consistent and practicable.

\section{Conclusion}

In this paper, we have represented the T2FSS in the most straightforward form to augment the soft-set theories. We also have exhibited some pictorial examples on the T2FSS. Three definitions, namely mid- $\alpha$-threshold fuzzy set, mid- $\lambda$ threshold fuzzy set, and the level fuzzy soft set, have been proposed. Further, we have proposed two algorithms, and at last, we have applied these algorithms to solve T2FSS and WT2FSS based on DMP. Many models are introduced by integrating a fuzzy set with other mathematical tools to capture more uncertainty. Type 2 fuzzy soft set is one of them. In the future, a new mathematical model can be introduced by using our proposed most straightforward representation of type 2 fuzzy soft set, which can open an original path to solving a decision making problem.

Acknowledgements The authors are thankful to the referees for some constructive comments.

Funding Not applicable.

Availability of data and material Not applicable.

\section{Compliance with ethical standards}

Conflict of interest The authors declare that there is no conflict of interest.

Open Access This article is licensed under a Creative Commons Attribution 4.0 International License, which permits use, sharing, adaptation, distribution and reproduction in any medium or format, as long as you give appropriate credit to the original author(s) and the source, provide a link to the Creative Commons licence, and indicate if changes were made. The images or other third party material in this article are included in the article's Creative Commons licence, unless indicated otherwise in a credit line to the material. If material is not included in the article's Creative Commons licence and your intended use is not permitted by statutory regulation or exceeds the permitted use, you will need to obtain permission directly from the copyright holder. To view a copy of this licence, visit http://creativecomm ons.org/licenses/by/4.0/.

\section{References}

1. Molodtsov D (1999) Soft set theory-1st result. Comput Math Appl 37:19-31

2. Zadeh LA (1965) Fuzzy sets. Inf Control 8:338-353. https://doi. org/10.1016/S0019-9958(65)90241-X
3. Kong Z, Gao L, Wang L, Li S (2008) The normal parameter reduction of soft sets and its algorithm. Comput Math Appl 56(12):3029-3037

4. Ali MI, Feng F, Liu X, Min WK, Shabir M (2009) On some new operations in soft set theory. Comput Math Appl 57(9):1547-1553

5. Zou Y, Xiao Z (2008) Data analysis approaches of soft sets under incomplete information. Knowl-Based Syst 21(8):941-945

6. Cagman N, Enginoglu S (2010) Soft set theory and uni-int decision making. Eur J Oper Res 207(2):848-855

7. Herawan T, Deris MM (2011) A soft set approach for association rules mining. Knowl-Based Syst 24(1):186-195

8. Gong K, Xiao Z, Zhang X (2010) The bijective soft set with its operations. Comput Math Appl 60(8):2270-2278

9. Basu TM, Mahapatra NK, Mondal SK (2012) A balanced solution of medical science. Appl Soft Comput 12:3260-3275

10. Paik B, Mondal SK (2019) A distance-similarity method to solve fuzzy sets and fuzzy soft sets based decision-making problems. Soft Comput. https://doi.org/10.1007/s00500-019-04273-z

11. Basu TM, Mondal SK (2015) A new solution approach using intuitionistic fuzzy cognitive map to solve intuitionistic fuzzy multi-criteria ranking problems in medical science. J Fuzzy Math $23: 431-442$

12. Manna S, Basu TM, Mondal SK (2019) Generalized trapezoidal intuitionistic fuzzy soft sets in risk analysis. Int J Appl Comput Math 5:66

13. Basu TM, Mondal SK (2017) Multi-criteria assignment techniques in multi-dimensional neutrosophic soft set theory. Neutrosophic Sets Syst 15:49-59

14. Basu TM, Mondal SK (2015) Neutrosophic soft matrix and its application in solving group decision making problems from medical science. Infinite Study. https://doi.org/10.5281/zenddo.23095

15. Xu W, Ma J, Wang S, Hao G (2010) Vague soft sets and their properties. Comput Math Appl 59(2):787-794

16. Feng F, Li C, Davvaz B, Ali MI (2010) Soft sets combined with fuzzy sets and rough sets: a tentative approach. Soft Comput 14(9):899-911

17. Manna S, Basu TM, Mondal SK (2018) Trapezoidal interval type-2 fuzzy soft stochastic set and its application in stochastic multicriteria decision-making. Granul comput. https://doi.org/10.1007/ s41066-018-0119-0

18. Manna S, Basu TM, Mondal SK (2019) A new algorithmic approach to linguistic valued soft multi-criteria group decision making problems using linguistic scale function. App Soft Comput. https://doi.org/10.1016/j.0505c.2019.105651

19. Maji PK, Roy AR, Biswas R (2004) On intuitionistic fuzzy soft sets. J Fuzzy Math 12(3):669-683

20. Atanassov KT (1986) Intuitionistic fuzzy sets. Fuzzy Sets Syst 20(1):87-96

21. Jiang Y, Tang Y, Chen Q, Liu H, Tang J (2010) Interval-valued intuitionistic fuzzy soft sets and their properties. Comput Math Appl 60(3):906-918

22. Jammalamadaka S, Qiu J, Ning N (2019) Predicting a stock portfolio with the multivariate Bayesian structural time series model: do news or emotions matter? Int J Artif Intell 17:81-104

23. Pozna C, Precup R (2014) Applications of signatures to expert systems modelling. Acta Polytechnica Hungarica 11(2):21-39

24. Zadeh LA (1975) The concept of a linguistic variable and its application to approximate reasoning. Inf Sci 8:199-249

25. Karnik NN, Mendel JM (2001) Operations on type-2 fuzzy sets. Fuzzy Sets Syst 122(2):327-348

26. Karnik NN, Mendel JM (1999) Applications of type-2 fuzzy logic systems to forecasting of timeseries. Inf Sci 120(1):89-111

27. Mendel JM, John RI, Liu F (2001) Interval type-2 fuzzy logic systems made simple. IEEE Trans Fuzzy Syst 122:327-348

28. Mitchell HB (2005) Pattern recognition using type-II fuzzy sets. Inf Sci 170(2-4):409-418 
29. Mizumoto M, Tanaka K (1981) Fuzzy sets of type-2 under algebraic product and algebraic sum. Fuzzy Sets Syst 5(3):277-290

30. Wu H, Wu Y, Luo J (2009) An interval type-2 fuzzy rough set model for attribute reduction. IEEE Trans Fuzzy Syst 17(2):301-315

31. Zeng J, Liu ZQ (2006) Type-2 fuzzy hidden Markov models and their application to speech recognition. IEEE Trans Fuzzy Syst 14(3):454-467

32. Mendel JM (2000) Uncertainty, fuzzy logic, and signal processing. Signal Process J 80:913-933. https://doi.org/10.1016/S01651684(00)00011-6

33. Castillo O, Melin P (2008) Type-2 fuzzy logic: theory and applications. Springer-Verlag, Heidelberg. https://doi.org/10.1109/GrC. 2007.118

34. Karnik NN, Mendel JM (1998) An introduction to type-2 fuzzy logic systems, technical report. University of Southern California, California. https://doi.org/10.1109/FUZZY.1998.686240

35. Hagras HA (2004) A hierarchical type-2 fuzzy logic control architecture for autonomous mobile robots. IEEE Trans Fuzzy Syst 12(4):524-539. https://doi.org/10.1109/TFUZZ.2004.832538

36. Mendel J (2015) Type-2 fuzzy sets and systems: a retrospective. Informatik Spektrum 38:523-532. https://doi.org/10.1007/ s00287-015-0927-4
37. Lathamaheswari M, Nagarajan D, Kavikumar J et al (2020) Triangular interval type-2 fuzzy soft set and its application. Complex Intell Syst 6:531-544. https://doi.org/10.1007/s40747-02000151-6

38. Melin P, Castillo O (2014) A review on type-2 fuzzy logic applications in clustering, classification and pattern recognition. Appl Soft Comput 21:568-577. https://doi.org/10.1016/j.asoc.2014.04. 017

39. Zhang Z and Zhang S (2012) Type-2 fuzzy soft sets and their applications in decision making. J Appl Math 2012:608681

40. Feng F, Jun YB, Liu X, Li L (2009) An adjustable approach to fuzzy soft set based decision making. J Comput Appl Math. https://doi. org/10.1016/j.cam.2009.11.055

41. Kong PZ, Gao LQ, Wang LF (2009) Comment on a fuzzy soft set theoretic approach to decision making problems. J Comput Appl Math 223:540-542

42. Roy AR, Maji PK (2007) A fuzzy soft set theoretic approach to decision making problems. J Comput Appl Math 203:412-418

Publisher's Note Springer Nature remains neutral with regard to jurisdictional claims in published maps and institutional affiliations. 